\title{
A Phylogenetic Independent Contrast Method under the Ornstein-Uhlenbeck Model and its Applications in Correlated Evolution
}

\author{
Cong Liang ${ }^{1, *}$ And YinguUn Deng ${ }^{1}$ \\ ${ }^{1}$ Center for Applied Mathematics, Tianjin University, Tianjin, 300192, P.R.China \\ *Correspondence to: cong.liang@tju.edu.cn
}

\begin{abstract}
1 Phylogenetic comparative methods are essential in studying the evolution of traits across a 2 phylogeny. Felsenstein's phylogenetic independent contrast (PIC) method and the 3 generalized least squares (GLS) regression were often utilized to study whether

4 evolutionary changes between traits were correlated. However, a neutral Brownian model is 5 assumed in the PIC method, which impacts the performance of the PIC method when the 6 trait is subject to adaptation. In recent years, the Ornstein-Uhlenbeck (OU) model has 7 attracted increasing attention in studying the evolution of traits with stabilizing selection.

8 In this study, we extended Felsenstein's PIC method under the OU model, which we 9 termed OU-PIC. We simulated trait evolution under the OU model on phylogenetic trees

with 8,10 , and 55 species. Compared to the PIC method, the OU-PIC method with correct stabilizing selection parameters achieved an appropriate type I error rate, the highest test power, and the lowest mean squared error. We presented a concise proof of the intrinsic connection between the OU-PIC and the generalized least squares (GLS) regression method in evaluating correlated evolution under the OU model. The OU-PIC method has a broad range of applications when trait evolution could be sufficiently modeled by the OU process. Compared with other phylogenetic comparative methods, OU-PIC avoids the inverse of the covariance matrix and would facilitate the analysis of
\end{abstract}


correlated evolution on large phylogenies.

Key words: Ornstein-Uhlenbeck model; phylogenetic independent contrasts; correlated evolution; generalized least squares

(1)

(1)

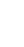

Correlated evolution studies the relationships between traits or between traits and their environments (Mark Pagel, 1999). By investigating correlated patterns in evolutionary changes, probable causal pathways of trait evolution might be inferred, for example in Podos (2001) and Böhmer et al. (2019). In these studies, traits were measured in species from clades of interest. But species cannot be regarded as independent samples in most instances because their evolutionary histories overlap hierarchically. Therefore, phylogenetically based methods are essential in analyzing such comparative data to ensure the accuracy and reliability of relevant hypothesis testing and inferences (Rohlf, 2006). Felsenstein's phylogenetic independent contrast (PIC) method (Felsenstein, 1985) has been widely utilized to correct the non-independence of species. The PIC method requires knowledge of the phylogeny with branch lengths and assumes a Brownian motion (BM) model of evolution. The phylogenetic independent contrasts returned by the method (also referred to as PICs, or contrasts) are independent of each other under the BM assumption, and hence are considered suitable for further statistical analysis. Another frequently adopted method for correlated evolution is the generalized least squares (GLS) regression (Martins and Hansen, 1997). The prediction power of one trait on another is evaluated in the GLS regression model. The covariance structure of the error terms describes the relationship among species, and is determined by the phylogeny and the evolution model. The BM assumption is also frequently adopted in the GLS method. Under this assumption, the PIC and the GLS method are intrinsically connected. The slope 
parameter of the ordinary least squares regression of PIC conducted through the origin is equivalent to the slope parameter of the GLS parameter in evaluating correlated evolution (Rohlf, 2006; Blomberg et al., 2012).

The Brownian model of evolution is neutral and has no adaptation term. In practice, the BM assumption of trait evolution is likely violated. Multiple simulation studies have evaluated the performance of different phylogenetic comparative methods (PCMs) when the underlying evolutionary process deviates from BM (Martins and Garland, 1991; Martins et al., 2002). Among these methods, the PIC method has been shown to outperform others in most cases and is generally acceptable in studying correlated evolution even if the underlying evolution model is not BM (Martins and Garland, 1991). A widely utilized alternative to the BM model is the Ornstein-Uhlenbeck (OU) model, where both the effect of stabilizing selection as well as random drift were modeled (Hansen, 1997; Butler and King, 2004). In contrast to the BM model where the variance of traits increases without limit, the trait value tends to drift around its optimal level with a bounded variance under the OU model. This bounded property made OU the preferred model in many evolutionary scenarios, for example in describing the evolution of molecular traits like gene expression (Bedford and Hartl, 2009; Yang et al., 2019). However, the covariance structure of species is more complicated under the OU model (Hansen and Martins, 1996; Hansen, 1997). More and more analytical methods were established to study trait evolution under the OU model in recent years (Rohlfs et al., 2014; Khabbazian et al., 2016). Difficulties have also been addressed in studying evolutionary questions with OU models (Ho and Ané, 2013, 2014).

In this manuscript, we derived a generalization of Felsenstein's PIC method under the OU model of trait evolution, which we termed as the OU-PIC method. Our method calculates $n-1$ phylogenetic independent contrasts (OU-PICs) from observations in $n$ species according to their phylogenetic structure. We modeled the level of correlated evolution by the correlation coefficient between the stochastic terms of OU processes, 
which enabled us to construct explicit statistical tests and inferences on correlated evolution. We studied the correlated evolution of continuous traits under the OU model using the OU-PIC method. Our simulation results showed that OU-PIC improves the statistical performance of the PIC method under the OU model. When the correct model parameter is specified, OU-PIC delivers appropriate type I error rates, obtained the highest power of tests, and the lowest mean squared errors in estimations. Finally, we highlighted the intrinsic connection between the OU-PIC and the GLS regression method by analyzing the contrast coefficient matrix.

We consider the Ornstein-Uhlenbeck model for the evolution of a continuous trait $X$ (Hansen, 1997; Butler and King, 2004). The evolution process is described by the following stochastic differential equation,

$$
d X_{t}=\lambda\left(\mu-X_{t}\right) d t+\sigma d W_{t}
$$

$W_{t}$ is a Wiener process or standard Brownian motion. It has Gaussian increments and its variance is linear to time $t$. The stochastic term, $\sigma d W_{t}$, models the effect of random drift with strength parameter $\sigma$. The deterministic term, $\lambda\left(\mu-X_{t}\right) d t$, models the effect of stabilizing selection. Parameter $\lambda$ measures the strength of stabilizing selection and is always positive. With stabilizing selection, $X_{t}$ tends to drift towards its fitness optimum $\mu$. If $X_{t}$ deviates far from $\mu$, the character value will be pulled towards its optimum with a large slope. If $\lambda=0$, Equation (1) becomes the Brownian model.

Equation (1) has analytical solutions, which makes it a tractable model for studying trait evolution. Given an ancestral state $x_{0}, X_{t}$ follows a normal distribution, with known expectation and variance. 


$$
\begin{aligned}
& \mathbf{E}\left[X_{t} \mid X_{0}=x_{0}\right]=\mu+\left(x_{0}-\mu\right) e^{-\lambda t}, \\
& \operatorname{Var}\left[X_{t} \mid X_{0}=x_{0}\right]=\frac{\sigma^{2}}{2 \lambda}\left(1-e^{-2 \lambda t}\right) .
\end{aligned}
$$

${ }_{93}$ The expectation of $X_{t}$ decays or increases exponentially with time. $t_{1 / 2}=\frac{\ln 2}{\lambda}$, also called

the half-time, is when $\mathbf{E}\left[X_{t}\right]$ is halfway towards its optimal. For a trait evolving along a

known phylogeny, the proportion of the half-time to the time span of the phylogeny

describes how fast stabilizing selection pulls $X_{t}$ to its optimal relative to the time span of

the phylogeny. Take $L$ as the distance from the root to current species on the phylogeny.

We define $\kappa$ as the relative strength of stabilizing selection, which is the ratio of $L$ and $t_{1 / 2}$,

$$
\kappa=\frac{L}{t_{1 / 2}}=\frac{\lambda L}{\ln 2}
$$

If $X$ has evolved a very long time $\tau, X_{\tau}$ follows a normal distribution that is independent of $x_{0}$ and $\tau$,

$$
X_{\tau} \sim N\left(\mu, \frac{\sigma^{2}}{2 \lambda}\right), \quad \text { as } \tau \rightarrow \infty
$$

\section{Trait Evolution along a Phylogenetic Tree Under the OU Model}

To study the evolution of a trait $X$ along a known phylogeny (for example as in Figure 1), the time interval between the last common ancestor of all species $(t=0)$ and current time $\left(t=t_{0}\right)$ is generally considered. In fact, most traits, especially molecular traits such as gene expression levels, could only be observed at the current time, i.e., $t=t_{0}$. As a result, we drop the time index of trait values and denote its value on tip node $k$ of the phylogeny by random variable $X_{k}$; also denote the trait value on the root of the phylogeny by random variable $X_{0}$. We made several general assumptions in this study: (1) Branch lengths on the phylogeny are in common clock scale. (2) Only extant species are considered, so all tip nodes obtain the same distance to the root of the tree. (3) The optimal trait value has not changed in the course of evolution. (4) When the evolutionary history overlapped, the evolution of $X_{k}$ are the same process. They became independent 
after the speciation event.

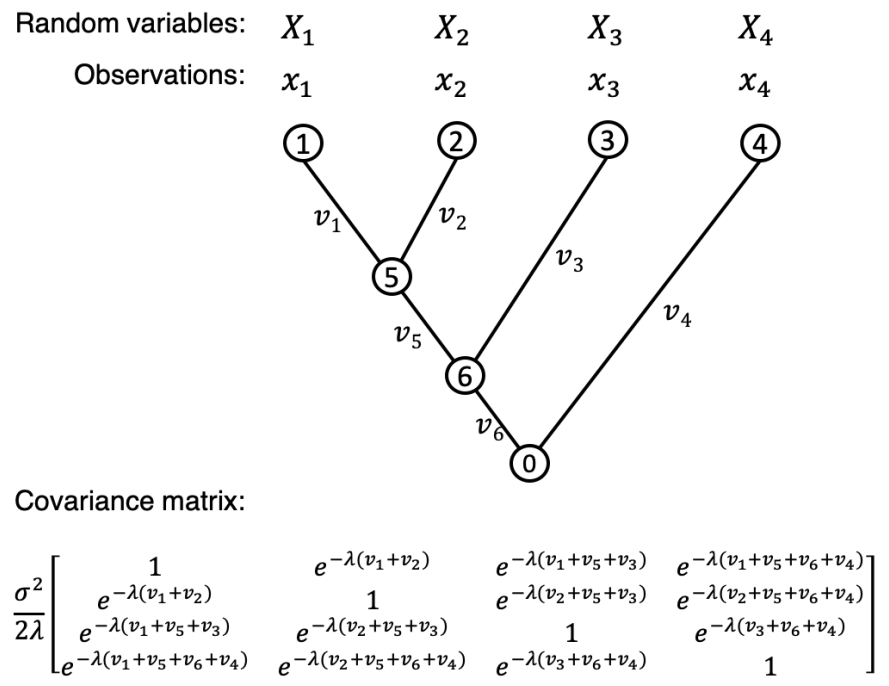

Figure 1. An illustration of a 4-species phylogeny and the evolution of trait $X$. Extant species are indexed from 1 to 4 , and the last common ancestor of all species is indexed as node 0 . Trait values are only observed in extant species, i.e., tips of the species tree. We used capitalized letters, $X_{1}, \cdots, X_{4}$ for random variables of trait $X$ in different species, and the corresponding lower letters, $x_{1}, \cdots, x_{4}$ for their observed values. When their evolutionary history overlapped, the evolution of $X_{k}$ are the same process, and processes in two species became independent after speciation. The branch lengths are in common clock scale and are given as $v_{i}$. The distance from the root to the tips of the species tree is a constant. We illustrated the covariance matrix of $\boldsymbol{X}=\left(X_{1}, \cdots, X_{4}\right)$ according to Equation (5).

Further, if trait $X$ originated long before the root of the phylogeny, the evolution time $\tau$ between the origin of $X$ and the root of the phylogeny is so long that we take $\tau \rightarrow \infty . X_{0}$ and all $X_{k}$ follow the stationary distribution in Equation (4) that $X_{0}, X_{k} \sim N\left(\mu, \frac{\sigma^{2}}{2 \lambda}\right)$. This ancient-origin assumption has been adopted in multiple studies and was also called the stationary assumption of the OU model (Hansen, 1997; Bedford and Hartl, 2009; Yang et al., 2019). We will discuss trait evolution under the OU model with this assumption throughout this manuscript.

As under the Brownian model, trait values on different nodes of the tree are correlated to each other under the OU model. The covariance between two nodes $i$ and $j$ could be derived using the analytical solution of OU processes (Appendix I, also in Hansen 
and Martins (1996)).

$$
\operatorname{Cov}\left(X_{i}, X_{j}\right)=\frac{\sigma^{2}}{2 \lambda} e^{-\lambda d(i, j)}
$$

$d(i, j)$ is the divergence time between $i$ and $j$. We illustrated a simple phylogenetic tree and the covariance matrix for its tip node values in Figure 1.

\section{Correlated Evolution under the OU Model}

We studied correlated evolution between two traits $X$ and $Y$ under the OU model (Liang et al., 2018). Both evolutionary trajectories follow the Ornstein-Uhlenbeck process:

$$
\begin{aligned}
& d X_{t}=\lambda_{x}\left(\mu_{x}-X_{t}\right) d t+\sigma_{x} d W_{t}^{\alpha} \\
& d Y_{t}=\lambda_{y}\left(\mu_{y}-Y_{t}\right) d t+\sigma_{y} d W_{t}^{\beta} .
\end{aligned}
$$

We introduced a parameter $\gamma_{x y}$ for the level of correlated evolution between $X$ and $Y$. It is the correlation between the two Wiener processes $W_{t}^{\alpha}$ and $W_{t}^{\beta}$ :

$$
\operatorname{Cov}\left(d W_{t}^{\alpha}, d W_{t}^{\beta}\right)=\gamma_{x y} d t
$$

Alternatively, $W_{t}^{\alpha}$ and $W_{t}^{\beta}$ could be considered as linear combinations of two independent Wiener processes $W_{t}^{\tilde{\alpha}}$ and $W_{t}^{\tilde{\beta}}$.

$$
\begin{aligned}
& d W_{t}^{\alpha}=d W_{t}^{\tilde{\alpha}} \\
& d W_{t}^{\beta}=\gamma_{x y} d W_{t}^{\tilde{\alpha}}+\sqrt{1-\gamma_{x y}^{2}} d W_{t}^{\tilde{\beta}} .
\end{aligned}
$$

According to this model, $\gamma_{x y} \in(-1,1) \cdot \gamma_{x y}=0$ indicates the evolution of $X$ and $Y$ are independent to each other; whereas $\gamma_{x y}$ close to -1 or 1 indicates strong correlation between the evolution of $X$ and $Y \cdot \gamma_{x y}= \pm 1$ are mathematically plausible but are biologically unrealistic. To address questions regarding correlated evolution between $X$ and $Y$, we investigate hypothesis tests and inferences with respect to parameter $\gamma_{x y}$.

We derive the covariance between $X_{i}$ (trait $X$ 's value on node $i$ ) and $Y_{j}$ (trait $Y$ 's value on node $j$ ) under the OU model with stationary assumption (Appendix II).

$$
\operatorname{Cov}\left(X_{i}, Y_{j}\right)=\gamma_{x y} \cdot \frac{\sigma_{x} \sigma_{y}}{\left(\lambda_{x}+\lambda_{y}\right)} \cdot e^{-\left(\lambda_{x} t_{i}+\lambda_{y} t_{j}\right)}
$$


Here, $t_{i}, t_{j}$ are the evolution time between $i, j$ and their last common ancestor $k$, respectively. The joint distribution of character values in $n$ extant species $\left(X_{1}, \cdots, X_{n}, Y_{1}, \cdots, Y_{n}\right)^{T}$ is multivariate normal with mean $\left(\mu_{x}, \cdots, \mu_{x}, \mu_{y}, \cdots, \mu_{y}\right)$ and elements in covariance matrix $\Sigma_{x y}$ as in Equation (9).

\section{Statistical Performance of OU-PICs in Evaluating Correlated Evolution}

We estimated the level of correlated evolution $\gamma_{x y}$ using the Pearson correlation coefficient between OU-PICs of two traits $X$ and $Y, \widehat{\gamma}_{x y}=r_{p i c}$ (see Results). According to Appendix III, $r_{p i c}$ is an unbiased estimator of $\gamma_{x y}$ when $\lambda_{x}=\lambda_{y}$ (or equivalently, $\kappa_{x}=\kappa_{y}$ ). We evaluated the statistical performance of $r_{p i c}$ in estimating $\gamma_{x y}$ with respect to type I error rates, the power of test, and the mean squared error.

Type I Error Rate We evaluated the type I error rates of using OU-PICs to test whether there is correlated evolution between two traits. The null hypothesis, $H_{0}: \gamma_{x y}=0$. The alternative hypothesis, $H_{1}: \gamma_{x y} \neq 0$. The type I error rate is the probability of rejecting the null hypothesis when it is in fact true. We simulated trait values with no correlated evolution $\left(\gamma_{x y}=0\right)$ under the OU model of evolution as well as the Brownian model. The level of relative stabilizing selection varies, $\kappa \in\left(2^{0}, 2^{1}, 2^{2}, 2^{3}, 2^{4}, 2^{5}\right) . \kappa$ were set the same for $X$ and $Y$ in each simulation. Theoretically, when $H_{0}$ is true, a transformation of the Pearson correlation coefficient, $t(r)=r \sqrt{\frac{m-2}{1-r^{2}}}$ follows Student's $t$-distribution with degrees of freedom $d f=m-2 . m$ here is the number of samples. When applying the PIC or OU-PIC method, $m$ equals to the number of contrasts. For a $n$-species phylogeny, $m=n-1$. When no comparative method was applied ("Raw Tips" in Fig. 5), $m$ equals to the number of species. We determined the two-sided critical values $t_{c}(\alpha)$ for varied $\alpha$ levels with $\mathbf{P}\left(|t|<t_{c}(\alpha)\right)=1-\alpha$, and then identified the corresponding critical values for Pearson correlation coefficients, $r_{c}(\alpha)$. The type I error rates were calculated by the fraction of observed $r_{p i c}$ beyond critical values, $\left|r_{p i c}\right|>r_{c}(\alpha)$. 
Power of Test We evaluated the power of the above hypothesis test when $H_{1}$ is true. We simulated the evolution of two traits $X$ and $Y$ with varied levels of correlated evolution, $\gamma_{x y} \in(0,0.25,0.5,0.75,0.9)$. The level of stabilizing selection also varies, $\kappa \in\left(0,2^{0}, 2^{1} \cdot 2^{2}, 2^{3}, 2^{4}, 2^{5}\right)$. The critical values $r_{c}(\alpha)$ of the test were determined using the empirical distributions of $r_{p i c}$ when $H_{0}$ is true; $\mathbf{P}_{H_{0}}\left(\left|r_{p i c}\right| \leqslant r_{c}(\alpha)\right)=1-\alpha$. The power of test was then estimated by the fraction of $r_{p i c}$ beyond the critical values when $H_{1}$ is true; power $=\mathbf{P}_{H_{1}}\left(|r|>r_{c}(\alpha)\right)$.

Mean Squared Error We calculated the mean squared error in estimating $\gamma_{x y}$ using $r_{p i c}$ with $M S E=\sum\left(r_{p i c}-\gamma_{x y}\right)^{2}$.

\section{Phylogenetic Trees Analyzed in This Study}

We tested OU-PICs on phylogenetic trees with a varied number of species and structures as illustrated in Figure 2. (1) An 8-species tree of Darwin's finches (Finch Tree). This is one of the four phylogenetic hypotheses adopted in Podos (2001) to study the correlated evolution of morphology and vocal signal structures in Darwin's finches. Branch lengths in this tree are arbitrary, and not necessarily in time scales. We chose this tree as a representation of small and relatively balanced phylogenies. (2) A 10-species tree containing 9 mammalian species and one bird (Brawand Tree). This is the species sampling tree in Brawand et al. (2011) to study the evolution of gene expression in mammalian organs. The study sampled broadly among mammals but also has a special focus on great apes. We chose this tree because Brawand et al. (2011) is one of the most frequently utilized resources to study transcriptome evolution, plus this tree has a special comb-like structure with highly varied branch lengths. (3) A 55-species tree of maples (genus Acer, Acer Tree). The correlated evolution of leaf size, sapling canopy allometry, and other related traits of Acer species have been studied in Ackerly and Donoghue (1998). We chose this tree to represent larger phylogenies. The time tree of Acer species were downloaded 
from the time tree of life project (http://www.timetree.org/) (Hedges et al., 2015).

Ambiguous nodes were removed manually from this tree in this study. We note that our OU-PIC method can also handle hard polytomy by setting some branch lengths as zero.

We simulated values of two traits in extant species on these trees by direct sampling from their joint multivariate distributions. The number of simulations $N=10,000$.

A

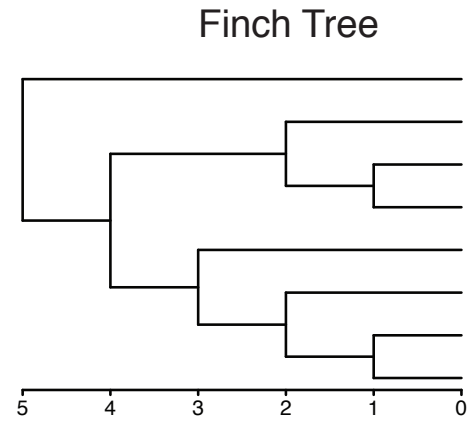

B

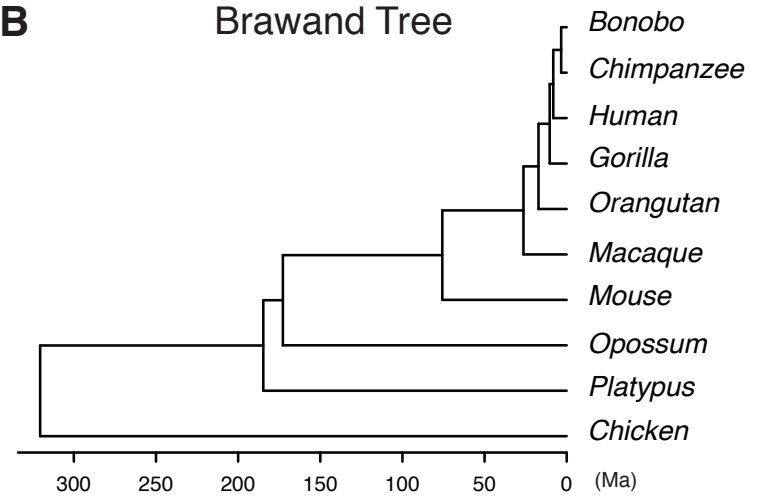

C. olivacea

C. parvulus

C. psittacula

C. pallida

G. scandens

G. fuliginosa

G. fortis

G. magniorstris

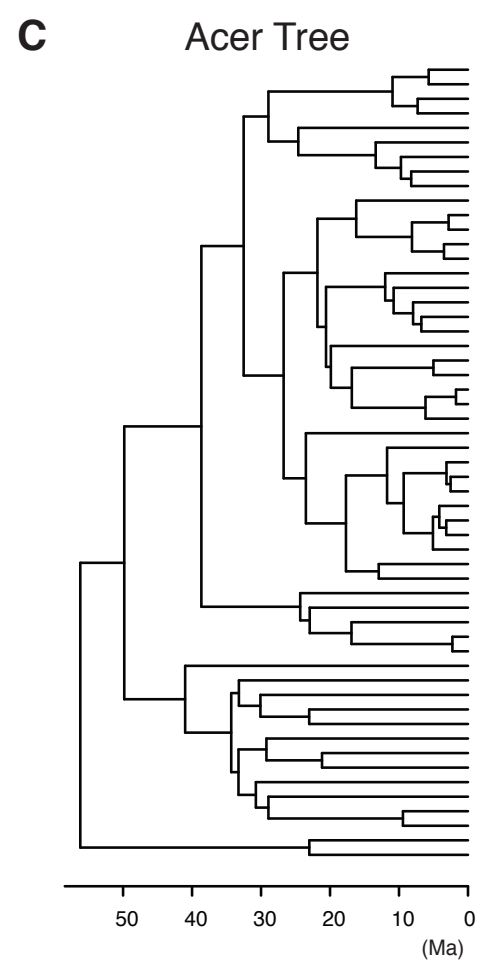

Figure 2. Phylogenetic trees analyzed in this study. (A) An 8-species tree of Darwin's finches studied in Podos (2001) (Finch Tree). The branch lengths are arbitrary. (B) A 10-species tree containing nine mammals and one brid (Brawand Tree). This tree was sampled in Brawand et al. (2011) to study the evolution of gene expression in mammals with a focus on great apes. This tree has a comb-like structure, and its branch lengths vary heavily. (C) A 55-species tree of Acer species studied in Ackerly and Donoghue (1998) (Acer Tree). Ambiguous nodes were manually removed from this tree in this analysis. Speciation times in (B) and (C) are extracted from the time tree of life project (http://www.timetree.org/) (Hedges et al., 2015). 
Results

\section{A Phylogenetic Independent Contrast Method for the OU model}

We adopted a tree pruning procedure analogous to Felsenstein's PIC method to calculate phylogenetic independent contrasts under the OU model (Algorithm 1). The algorithm traverses the internal nodes of a species tree according to the post-order and prunes their descendants recursively (Fig. 3). The post-order traversal is often used to delete a tree since the ancestor node is visited after its descendants in this procedure. In each iteration of Algorithm 1, two descendants of an internal node $w_{i}$ were removed, making $\mathrm{w}_{i}$ a new leaf of the pruned tree. We denoted the two descendants of $\mathrm{w}_{i}$ by $\mathrm{x}_{l}$ and $\mathrm{x}_{r}$, let $x_{l}$ and $x_{r}$ denote their trait values, and let $v_{l}, v_{r}$ denote their distances to $\mathrm{w}_{i}$. Then, we calculated a contrast $c_{i}$, which is a weighted difference between $x_{l}$ and $x_{r}$, and an intermediate value $w_{i}$, which is a weighted average of $x_{l}$ and $x_{r}$.

$$
\begin{aligned}
& c_{i}=\eta_{i} e^{-\lambda v_{r}} x_{l}-\eta_{i} e^{-\lambda v_{l}} x_{r}, \\
& w_{i}=a_{i} x_{l}+b_{i} x_{r} .
\end{aligned}
$$

Here, $e^{-\lambda v_{r}}$ and $-e^{-\lambda v_{l}}$ make the mean value of the contrast zero. $\eta_{i}$ is the normalizing factor that makes the variances of all contrasts consistent. $a_{i}$ and $b_{i}$ were determined by the condition that the covariance between the contrast and the intermediate value is zero, and that intermediate values have consistent covariances. $c_{i}$ is the $i$-th contrast returned by the algorithm. $w_{i}$ is assigned to node $\mathbf{w}_{i}$ in the pruned tree. At the end of each iteration, we modified the branch length between $\mathrm{w}_{i}$ and its nearest ancestor by $\Delta d\left(\mathrm{w}_{i}, \mathrm{w}_{i}^{\text {anc }}\right)$ to make the covariance matrix among leaf nodes maintain the same structure in the pruned tree. These processes were iterated until there is only one node left on the pruned tree.

We specified the formulas of $\eta_{i}, a_{i}, b_{i}$ and $\Delta d\left(\mathrm{w}_{i}, \mathrm{w}_{i}^{\text {anc }}\right)$ in Equation (23) (Appendix III). Our algorithm assumed that the stabilizing selection parameter $\lambda$ is known along with the species tree. In practice, $\lambda$ could be estimated using maximum likelihood or other methods designed for the relevant data type (Bedford and Hartl, 2009; Gu et al., 2019). 


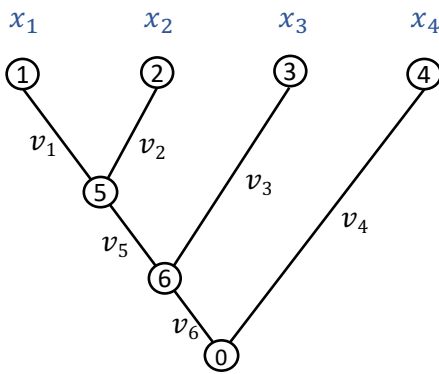

Tree 1

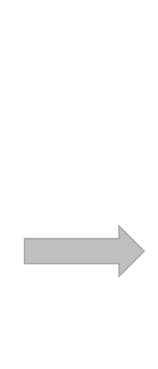

$x_{4}$

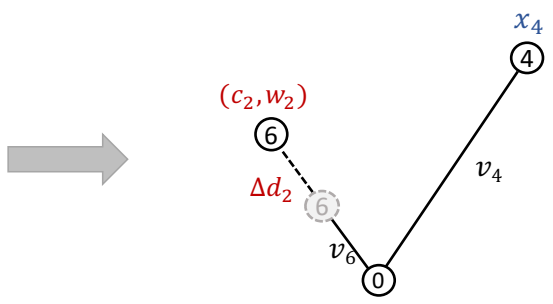

Tree 3

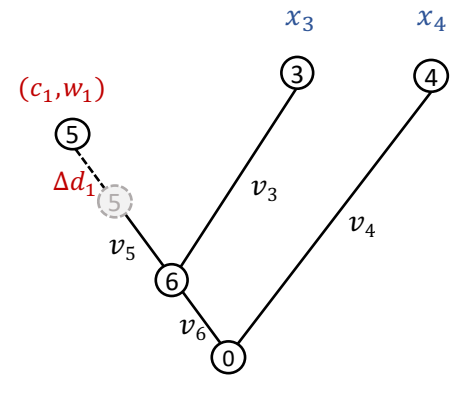

Tree 2

(0)

Tree 4

Figure 3. An illustration of the tree pruning process to calculate OU-PICs following Algorithm 1. The procedure is analogous to the tree pruning procedure in Felsenstein's PIC method. Internal nodes were visited by the order $5 \rightarrow 6 \rightarrow 0$. In each iteration, two leaf nodes were trimmed and their last common ancestor became a leaf in the pruned tree. A contrast $c_{i}$ and an intermediate node value $w_{i}$ were calculated in each iteration. $c_{i}$ is returned as the $i$-th contrast by the algorithm. $w_{i}$ is assigned to the new leaf node in the pruned tree. A modification of branch length $\Delta d_{i}$ is calculated between the new leaf node and its nearest ancestor. The process continues until only one node remained. A total of $n-1$ contrasts and intermediate values were calculated from this procedure.

Our algorithm returns $n-1$ contrasts (OU-PICs) for traits observed in $n$ extant species. To describe the statistical characteristics of our method, we distinguish random variables from their observed values in notations. We use capitalized letters to indicate random variables and the corresponding lowercase letters for observed or calculated values. For example, $C_{i}$ stands for an independent contrast random variable, whereas $c_{i}$ represents a contrast calculated from observed trait values. We showed that the OU-PICs have the following properties (proof in Appendix III):

1. $C_{i}$ are independent and identically distributed following a normal distribution; $C_{i} \sim N(0, \phi)$, the normalized variance $\phi=\frac{\sigma^{2}}{\lambda}$

2. The contrasts of two traits $X$ and $Y$ are pairwise correlated and their correlation equals the correlated evolution parameter $\gamma_{x y}$, if they obtain the same level of stabilizing selection, $\lambda_{x}=\lambda_{y}$. 
Like Felsenstein's PIC method, our OU-PIC method removes the correlation among species resulted from overlapping evolutionary histories. For two traits $X$ and $Y$ with the same level of stabilizing selection on a known phylogeny, the Pearson correlation coefficient between their OU-PICs is an unbiased estimator of their level of correlated evolution $\gamma_{x y}$.

\section{Algorithm 1 Phylogenetic Independent Contrast Method under the OU Model Require:}

1. A species tree with branch length in time scale;

2. Trait values on extant species $x_{1}, x_{2}, \cdots, x_{n}$;

3. The stabilizing selection parameter $\lambda$ for trait $X$.

Ensure: $\quad n-1$ independent contrasts $c_{1}, \cdots, c_{n-1}$.

1: while post-order traverse inner node $\mathbf{w}_{i}$ do

2: $\quad$ Denote the left and right child of inner node $\mathrm{w}_{i}$ as $\mathrm{x}_{l}$ and $\mathrm{x}_{r}$, with trait values $x_{l}$ and $x_{r} ;$

3: $\quad v_{l} \leftarrow d^{(i)}\left(\mathrm{w}_{i}, \mathrm{x}_{l}\right)$ and $v_{r} \leftarrow d^{(i)}\left(\mathrm{w}_{i}, \mathrm{x}_{r}\right)$, $d^{(i)}$ is the distance function between two nodes in the $i$-th tree;

4: $\quad c_{i} \leftarrow \eta_{i} e^{-\lambda v_{r}} x_{l}-\eta_{i} e^{-\lambda v_{l}} x_{r}$, $\eta_{i}$ is a normalizing factor such that $\operatorname{Var}\left(C_{i}\right)=\frac{\sigma^{2}}{\lambda}$;

5: $\quad w_{i} \leftarrow a_{i} x_{l}+b_{i} x_{r}$, $a_{i}$ and $b_{i}$ were chosen such that $\operatorname{Cov}\left(C_{i}, W_{i}\right)=0$ and that $\operatorname{Var}\left(W_{i}\right)=\frac{\sigma^{2}}{2 \lambda}$;

6: Prune node $\mathrm{x}_{l}$ and $\mathrm{x}_{r}$, then assign $w_{i}$ to node $\mathbf{w}_{i}$ on the $(i+1)$-th tree;

7: $\quad d^{(i+1)}\left(\mathrm{w}_{i}, \mathrm{w}_{i}^{\text {anc }}\right) \leftarrow d^{(i)}\left(\mathrm{w}_{i}, \mathrm{w}_{i}^{\text {anc }}\right)+\Delta d\left(\mathrm{w}_{i}, \mathrm{w}_{i}^{\text {anc }}\right)$, where $e^{-\lambda \Delta d\left(\mathrm{w}_{i}, \mathbf{w}_{i}^{a n c}\right)}=a_{i} e^{-\lambda v_{l}}+b_{i} e^{-\lambda v_{r}}, \mathbf{w}_{i}^{a n c}$ is the most recent ancestor of $\mathbf{w}_{i}$;

8: $\quad i \leftarrow i+1$

9: end while

10: return all $c_{i}, i \in(1, n-1)$. 
The Coefficient Matrix of OU-PICs

According to Algorithm 1, OU-PICs are linear combinations of observed trait values. We rewrote OU-PICs as $c_{i}=\boldsymbol{p}_{i}^{T} \boldsymbol{x}$. Here, $\boldsymbol{x}=\left(x_{1}, \cdots, x_{n}\right)^{T}$ are observed trait values in extant species. $\boldsymbol{p}_{i}(i=1, \cdots, n-1)$ are contrast coefficient vectors each with $n$ elements. Bold faces denote column vectors throughout this paper. Let $\boldsymbol{c}=\left(c_{1}, \cdots, c_{n-1}\right)^{T}$ denote the contrast vector. We have,

$$
\boldsymbol{c}=P^{T} \boldsymbol{x}, \quad P=\left[\begin{array}{ccc}
\mid & & \mid \\
\boldsymbol{p}_{\boldsymbol{1}}, & \cdots, & \boldsymbol{p}_{\boldsymbol{n}-\boldsymbol{1}} \\
\mid & & \mid
\end{array}\right] .
$$

We call $P$ the contrast coefficient matrix. Knowing that the contrasts are independent identically distributed random variables, the covariance matrix of the contrast vector is diagonalized. We have,

$$
\operatorname{Cov}(\boldsymbol{c})=P^{T} \Sigma P=\phi I_{n-1}
$$

Here, $I_{n-1}$ is an order $n-1$ identity matrix. In fact, Equation (12) is also true for Felsenstein's PICs. Both PICs and OU-PICs are linear transformations of trait values such that the covariance matrix is diagonalized under given evolutionary assumptions. The OU-PIC method is a generalization of Felsenstein's PIC method to OU assumptions.

The contrast coefficient matrix of OU-PICs differs from that of PICs in its non-zero terms. Under the BM model, accumulated variances in traits increase linearly with evolution time without limit. Whereas under the OU model, the variances of traits are constrained by stabilizing selection. Therefore, contrasts associated with long branches tend to be normalized with a greater variance in the PIC method than in the OU-PIC method. To compare the strength of stabilizing selection across species trees, we defined a dimension one quantity, $\kappa$, which describes how fast stabilizing selection drags the trait value towards its optimal relative to the time span of the phylogeny. Specifically, $\kappa$ is proportional to the product of $\lambda$ and the distance between the root and tips of the species tree (see Methods). 

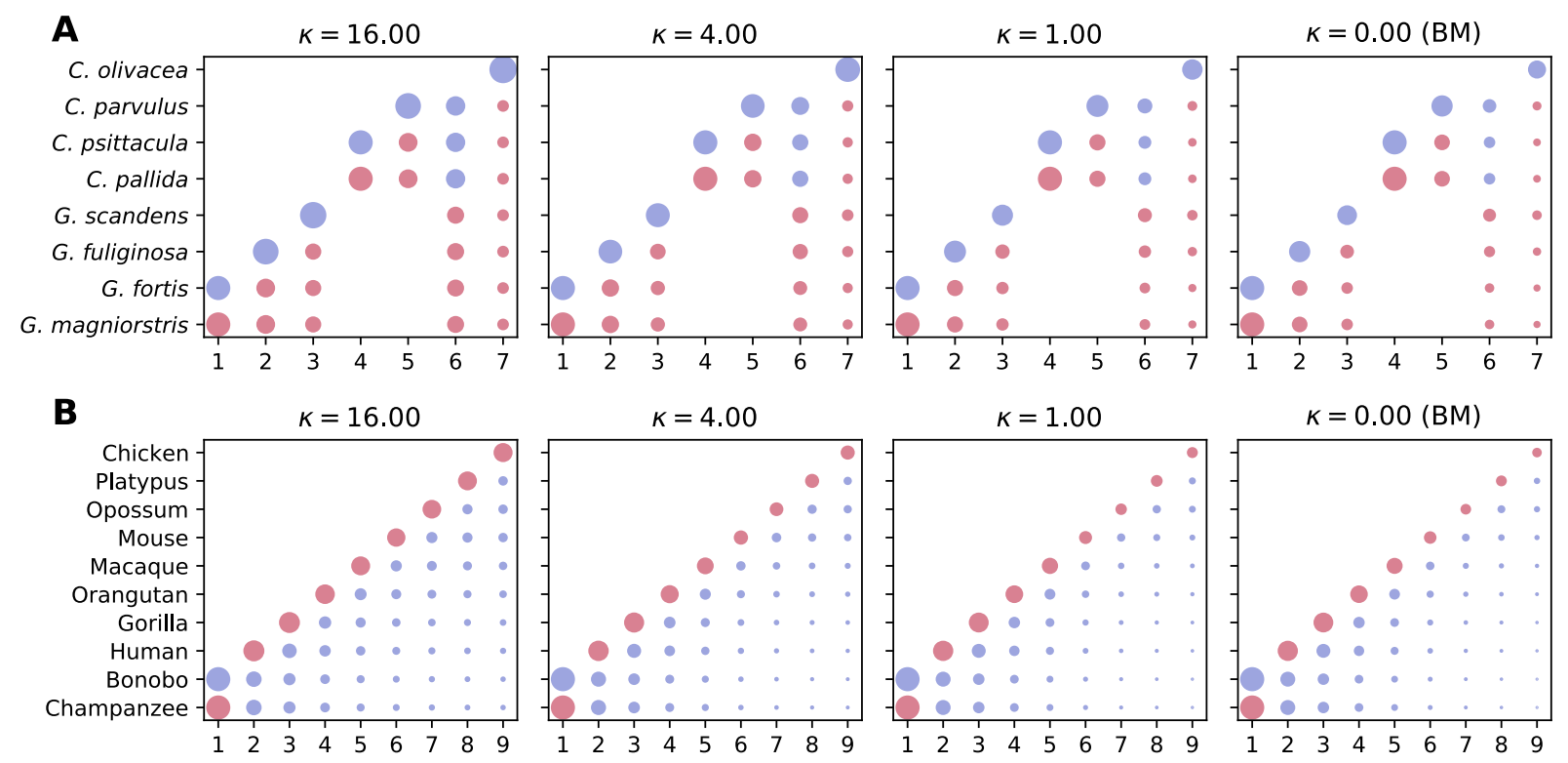

Figure 4. Visualization of the coefficient matrix of OU-PICs and PICs for (A) the Finch Tree, and (B) the Brawand Tree. The level of relative stabilizing selection $\kappa$ varies under the OU model. When $\kappa=0$, the evolution model is $\mathrm{BM}$, and the coefficient matrix of PICs is plotted. The horizontal axis in each panel represents the index of contrasts. The vertical axis in each panel represents extant species on a species tree. To compare coefficients for different $\kappa$, coefficients were normalized according to the coefficients of contrast 1 in each panel (the bottom left value). The size of the points is proportional to the absolute value of the normalized coefficients. The color of points represents the sign of coefficients, red for positive, blue for negative. We observed distinct coefficients for PICs and OU-PICs in contrasts associated with long branches, for example contrasts 3 and 7 in the Finch Tree, and contrasts 7-9 in the Brawand Tree. The difference between coefficients of PICs and OU-PICs increases with $\kappa$.

We observed shrunken coefficients of PICs associated with long branches compared to that of OU-PICs (Fig. 4 and Supp. Fig. 1). When the relative stabilizing selection is moderate or strong, for instance $\kappa>10$ for the Finch tree and the Brawand tree, the coefficients of OU-PICs deviate from the coefficients of PICs substantially. As $\kappa$ decreases, the differences between the coefficients of OU-PICs and the coefficients of PICs decline. When the relative stabilizing selection is weak, for instance $\kappa \leqslant 1$ for the Finch tree and the Brawand tree, the coefficients of OU-PICs are close to that of PICs. In this case, the evolution process of traits on the species tree can be approximated by a BM process. We also observed that the differences between the coefficients of OU-PICs and PICs are more significant in the Brawand tree than in the Finch tree, because branch lengths vary more 
heavily in the Brawand tree.

\section{Evaluating Correlated Evolution Using OU-PICs}

To evaluate the statistical performance of OU-PICs in studying correlated evolution, we simulated the evolution of two traits $X$ and $Y$ with varied levels of correlated evolution and stabilizing selection. The strength of stabilizing selection for two traits were set equal in the simulation, $\kappa_{x}=\kappa_{y}=\kappa$. The contrasts were calculated using varied assumed levels of stabilizing selection $(\widehat{\kappa})$. When $\widehat{\kappa}>0$, the OU-PIC method was applied to get the contrasts. When $\widehat{\kappa}=0$, the PIC method is utilized. The level of correlated evolution was then estimated by the Pearson correlation coefficient between the contrasts of two traits, $\widehat{\gamma}_{x y}=r_{p i c}(\widehat{\kappa})$. We compared the statistical performance of the PIC and the OU-PIC method with respect to (1) the type I error rate when $\gamma_{x y}=0$; (2) the power of test when $\gamma_{x y}>0$; and (3) the mean squared error in estimating $\gamma_{x y}$ (see Methods).

We found that OU-PIC achieved appropriate type I error rates when $\widehat{\kappa}=\kappa$, i.e. the correct level of stabilizing selection was assumed in the analysis (Fig. 5). If $\widehat{\kappa}$ deviates from $\kappa$ by only several folds, the type I error was slightly inflated. For example, the type I error is always smaller than 0.06 for all three species trees when the difference between $\widehat{\kappa}$ and $\kappa$ is less than two folds and $\alpha=0.05$. However, if $\widehat{\kappa}$ deviates excessively from $\kappa$, type I error rates could be highly inflated. Notably, the type I error rate was inflated by more than two folds if the PIC is inappropriately adopted when the underlying stabilizing selection is strong. For example, the type I error $=0.136$ for the Brawand tree and 0.173 for the Acer tree when $\kappa=32, \widehat{\kappa}=0$ and $\alpha=0.05$. Similarly, we obtained the highest power of test (Fig. 6 and Supp. Fig. 2) and the smallest mean squared error in estimating $\gamma_{x y}$ (Supp. Fig. 3 and 4$)$ when the appropriate $\kappa$ is specified $(\widehat{\kappa}=\kappa)$. When comparing species trees, we found that the Brawand tree and the Acer tree are more sensitive to parameters and model assumptions than the smaller Finch tree. Overall, these results suggest that it is important to evaluate the impact of stabilizing selection in comparative studies. Methods 
should be adopted accordingly to achieve the desired statistical performance when evaluating correlated evolution. When $\kappa$ is small $(\kappa \ll 1)$, the BM model is sufficient to describe the underlying evolutionary process. However, as $\kappa$ increases $(\kappa>1)$, the BM model can no longer serve as an adequate model. The statistical performance of the PIC method drops significantly. Specifically designed methods such as the OU-PIC method could improve the statistical performance under such circumstances.

\begin{tabular}{|c|c|c|c|c|c|c|c|c|}
\hline $\mathbf{A}$ & & & Finch & Tree, $r$ & $=8, \alpha=$ & $=0.05$ & & \\
\hline$\kappa=32-$ & 0.0491 & 0.0496 & 0.0496 & 0.0499 & 0.0521 & 0.0590 & 0.0676 & 0.0822 \\
\hline$\kappa=16-$ & 0.0499 & 0.0503 & 0.0503 & 0.0506 & 0.0532 & 0.0604 & 0.0690 & 0.0831 \\
\hline$\kappa=8-$ & 0.0514 & 0.0507 & 0.0507 & 0.0503 & 0.0528 & 0.0583 & 0.0653 & 0.0781 \\
\hline$\kappa=4-$ & 0.0531 & 0.0518 & 0.0517 & 0.0504 & 0.0488 & 0.0514 & 0.0560 & 0.0659 \\
\hline$\kappa=2-$ & 0.0655 & 0.0612 & 0.0610 & 0.0585 & 0.0535 & 0.0507 & 0.0519 & 0.0577 \\
\hline$\kappa=1$ & 0.0777 & 0.0708 & 0.0705 & 0.0670 & 0.0588 & 0.0526 & 0.0506 & 0.0530 \\
\hline$\kappa=0$ & 0.1019 & 0.0891 & 0.0887 & 0.0840 & 0.0713 & 0.0592 & 0.0532 & 0.0505 \\
\hline & Raw Tips & $\widehat{k}=32$ & $\widehat{K}=16$ & $\widehat{K}=8$ & $\widehat{\kappa}=4$ & $\widehat{K}=2$ & $\widehat{\kappa}=1$ & $\widehat{K}=0$ \\
\hline $\mathbf{B}$ & & & Brawanc & d Tree, & $\eta=10$ & $\alpha=0.05$ & & \\
\hline$\kappa=32-$ & 0.0567 & 0.0493 & 0.0532 & 0.0669 & 0.0900 & 0.1095 & 0.1226 & 0.1357 \\
\hline$\kappa=16-$ & 0.0725 & 0.0528 & 0.0502 & 0.0550 & 0.0690 & 0.0854 & 0.0970 & 0.1101 \\
\hline$\kappa=$ & 0.0977 & 0.0633 & 0.0546 & 0.0498 & 0.0547 & 0.0660 & 0.0756 & 0.0882 \\
\hline$\kappa=4$ & 0.1336 & 0.0861 & 0.0700 & 0.0545 & 0.0489 & 0.0534 & 0.0592 & 0.0690 \\
\hline$\kappa=2$ & 0.1693 & 0.1151 & 0.0959 & 0.0726 & 0.0553 & 0.0500 & 0.0514 & 0.0584 \\
\hline$\kappa=1$ & 0.1973 & 0.1418 & 0.1213 & 0.0926 & 0.0645 & 0.0523 & 0.0498 & 0.0529 \\
\hline$\kappa=0$ & 0.2385 & 0.1827 & 0.1646 & 0.1311 & 0.0922 & 0.0643 & 0.0534 & 0.0497 \\
\hline & Raw Tips & $\widehat{k}=32$ & $\widehat{\kappa}=16$ & $\widehat{\kappa}=8$ & $\widehat{\kappa}=4$ & $\widehat{\kappa}=2$ & $\widehat{\kappa}=1$ & $\widehat{\kappa}=0$ \\
\hline & & & Acer T & ree, $n=$ & $=55, \alpha=$ & $=0.05$ & & \\
\hline$\kappa=32-$ & 0.0517 & 0.0503 & 0.0531 & 0.0677 & 0.1002 & 0.1321 & 0.1518 & 0.1733 \\
\hline$\kappa=16$ & 0.0543 & 0.0509 & 0.0487 & 0.0548 & 0.0750 & 0.0996 & 0.1160 & 0.1346 \\
\hline$\kappa=8-$ & 0.0698 & 0.0635 & 0.0542 & 0.0492 & 0.0568 & 0.0721 & 0.0838 & 0.0990 \\
\hline$\kappa=$ & 0.1059 & 0.0977 & 0.0822 & 0.0598 & 0.0491 & 0.0543 & 0.0620 & 0.0735 \\
\hline$\kappa=2$ & 0.1591 & 0.1502 & 0.1328 & 0.0968 & 0.0594 & 0.0506 & 0.0526 & 0.0592 \\
\hline$\kappa=1$ & 0.2067 & 0.1980 & 0.1818 & 0.1407 & 0.0778 & 0.0533 & 0.0504 & 0.0533 \\
\hline$\kappa=0-$ & 0.2818 & 0.2746 & 0.2633 & 0.2227 & 0.1283 & 0.0688 & 0.0543 & 0.0502 \\
\hline & Raw Tips & $\widehat{K}=32$ & $\widehat{K}=16$ & $\widehat{K}=8$ & $\widehat{K}=4$ & $\widehat{K}=2$ & $\widehat{K}=1$ & $\widehat{K}=0$ \\
\hline
\end{tabular}

Figure 5. Type I error rates in testing whether there is correlated evolution on (A) the Finch Tree, (B) the Brawand Tree, and (C) the Acer Tree. We simulated the evolution of two traits with no correlated evolution, and estimated the fraction of $\widehat{\gamma}_{x y}$ beyond the theoretical threshold for $\alpha=0.05$. The vertical axis in each panel is the true level of stabilizing selection in simulation, $\kappa$. The horizontal axis in each panel is the level of stabilizing selection used for calculating OU-PIC. The first column, "Raw Tips", correlated evolution is estimated with no comparative method, i.e. the correlation between observed values in extant species. When $\kappa=0$, the true evolution model is BM. When $\widehat{\kappa}=0$, PICs were utilized in the analysis. We observed appropriate type I errors when the correct level of correlated evolution is specified in the analysis, $\widehat{\kappa}=\kappa$ (diagonal of the table). Type I error rates were inflated when $\widehat{\kappa}$ deviate from $\kappa$. 
A

Finch Tree, $\mathrm{n}=8, \gamma=0.50$

\begin{tabular}{|c|c|c|c|c|c|c|c|}
\hline & & & & & & & \\
\hline$\kappa=32$ & 0.334 & 0.334 & 0.333 & 0.327 & 0.316 & 0.305 & 0.289 \\
\hline$\kappa=16$ & 0.327 & 0.328 & 0.328 & 0.323 & 0.311 & 0.300 & 0.283 \\
\hline$\kappa=$ & 0.329 & 0.329 & 0.330 & 0.329 & 0.320 & 0.308 & 0.295 \\
\hline$\kappa$ & 0.320 & 0.321 & 0.324 & 0.327 & 0.326 & 0.318 & 0.307 \\
\hline$k-$ & 0.314 & 0.314 & 0.320 & 0.329 & 0.332 & 0.330 & 0.320 \\
\hline$\kappa=$ & 0.296 & 0.297 & 0.300 & 0.313 & 0.322 & 0.326 & 0.322 \\
\hline$\kappa=0-$ & 0.283 & 0.283 & 0.287 & 0.300 & 0.315 & 0.324 & 0.325 \\
\hline & $\widehat{\kappa}={ }^{\prime} 32$ & $\widehat{K}=16$ & $\widehat{\kappa}=8$ & $\widehat{\kappa}=4$ & $\widehat{\kappa}=2$ & $\widehat{\kappa}=1$ & $\widehat{\kappa}=0$ \\
\hline
\end{tabular}

B

Brawand Tree, $\mathrm{n}=10, \gamma=0.50$

\begin{tabular}{|c|c|c|c|c|c|c|c|}
\hline$r=32-$ & 0.420 & 0.412 & 0.389 & 0.355 & 0.332 & 0.319 & 0.306 \\
\hline$=16$ & 0.407 & 0.414 & 0.403 & 0.379 & 0.357 & 0.341 & 0.326 \\
\hline$\kappa=$ & 0.391 & 0.411 & 0.424 & 0.411 & 0.389 & 0.371 & 0.353 \\
\hline$\kappa=4$ & 0.357 & 0.379 & 0.408 & 0.418 & 0.407 & 0.396 & 0.376 \\
\hline$\kappa=2$ & 0.323 & 0.344 & 0.378 & 0.409 & 0.416 & 0.412 & 0.398 \\
\hline$\kappa=$ & 0.296 & 0.315 & 0.345 & 0.382 & 0.410 & 0.416 & 0.409 \\
\hline \multirow[t]{2}{*}{$\kappa=0-$} & 0.272 & 0.285 & 0.310 & 0.346 & 0.384 & 0.406 & 0.418 \\
\hline & $\widehat{K}=32$ & $\widehat{K}=16$ & $\widehat{K}=8$ & $\widehat{k}=4$ & $\widehat{K}=$ & $\widehat{K}=$ & $\widehat{k}=$ \\
\hline
\end{tabular}

C

Acer Tree, $\mathrm{n}=55, \gamma=0.50$

\begin{tabular}{|c|c|c|c|c|c|c|c|}
\hline$K=32-$ & 0.989 & 0.988 & 0.981 & 0.959 & 0.926 & 0.898 & 0.869 \\
\hline$=16$ & 0.987 & 0.989 & 0.986 & 0.975 & 0.953 & 0.937 & 0.915 \\
\hline$\kappa=$ & 0.980 & 0.986 & 0.991 & 0.987 & 0.979 & 0.971 & 0.958 \\
\hline$\kappa=4$ & 0.957 & 0.969 & 0.982 & 0.987 & 0.985 & 0.980 & 0.975 \\
\hline$k=2$ & 0.897 & 0.917 & 0.956 & 0.984 & 0.990 & 0.989 & 0.984 \\
\hline$\kappa=$ & 0.834 & 0.857 & 0.903 & 0.970 & 0.986 & 0.989 & 0.987 \\
\hline \multirow[t]{2}{*}{$\kappa=0-$} & 0.724 & 0.739 & 0.793 & 0.918 & 0.979 & 0.988 & 0.990 \\
\hline & $\widehat{\kappa}=32$ & $\widehat{K}=16$ & $\widehat{K}=8$ & $\widehat{K}=4$ & $\widehat{K}=2$ & $\widehat{\kappa}=1$ & $\widehat{\kappa}=0$ \\
\hline
\end{tabular}

Figure 6. The power of test on (A) the Finch Tree, (B) the Brawand Tree, and (C) the Acer Tree given $\gamma=0.5$. The highest test power is achieved when the correct level of stabilizing selection is specified, i.e. $\widehat{\kappa}=\kappa$.

Correlated Evolution with Unequal Stabilizing Selection Parameters

When $\kappa_{x} \neq \kappa_{y}$, the covariance matrix of OU-PICs for $X$ and $Y$ becomes analytically complicated. Pairs of OU-PICs, $\left(C_{i}^{X}, C_{i}^{Y}\right)$ for $i=1,2, \cdots, n-1$, become inter-correlated in this case. As a result, we tested the performance of OU-PICs in evaluating correlated evolution when $\kappa_{x} \neq \kappa_{y}$ numerically. In practice, if $\kappa_{x}$ and $\kappa_{y}$ dramatically differ from each other (e.g. by 5 folds or more), the effect of stabilizing selection on their evolution processes also diverges significantly. It does not make much biological sense to study correlated evolution between such two traits. Therefore, we simulated trait evolution with slightly different $\kappa_{x}$ and $\kappa_{y}$, 
${ }_{306}\left(\kappa_{x}, \kappa_{y}\right)=(4.5,5.5),(3,7)$ or $(2,8)$. We again evaluated the type I error rates, the power of

${ }_{307}$ tests, and mean squared errors in studying the level of correlated evolution using the

${ }_{308}$ OU-PIC method (assuming $\widehat{\kappa}=\kappa_{x}, \kappa_{y}$, or $\left.\left(\kappa_{x}+\kappa_{y}\right) / 2\right)$ and the PIC method $(\widehat{\kappa}=0)$. We

${ }_{309}$ found that in all three phylogenetic trees we analyzed, the OU-PIC method still obtained

${ }_{310}$ appropriate statistical performance and outperformed the PIC method (Table 1).

Table 1. The type I error rates, test power, and mean squared errors using the PIC and OU-PIC method to evaluate correlated evolution when $\kappa_{x} \neq \kappa_{y}$.

\begin{tabular}{|c|c|c|c|c|c|c|c|c|c|c|c|c|c|c|c|c|}
\hline & \multirow[b]{2}{*}{$\kappa_{x}$} & \multirow{2}{*}{$\kappa_{y}$} & \multirow[b]{2}{*}{$\widehat{\kappa}$} & \multirow{2}{*}{$\begin{array}{c}\text { Type l error } \\
\text { rate }\end{array}$} & \multicolumn{4}{|c|}{$\gamma=0.25$} & \multicolumn{4}{|c|}{$\gamma=0.50$} & \multicolumn{4}{|c|}{$\gamma=0.75$} \\
\hline & & & & & power & mean & std & mse & power & mean & std & mse & power & mean & std & mse \\
\hline \multirow{12}{*}{ 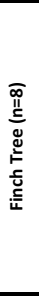 } & & & 4.5 & 0.051 & 0.133 & 0.230 & 0.389 & 0.152 & 0.328 & 0.466 & 0.337 & 0.115 & 0.696 & 0.715 & 0.230 & 0.054 \\
\hline & & & 5.5 & 0.049 & 0.133 & 0.231 & 0.390 & 0.153 & 0.322 & 0.465 & 0.336 & 0.114 & 0.697 & 0.716 & 0.230 & 0.054 \\
\hline & & & 5.0 & 0.049 & 0.137 & 0.231 & 0.390 & 0.153 & 0.330 & 0.466 & 0.337 & 0.115 & 0.700 & 0.716 & 0.228 & 0.053 \\
\hline & 4.5 & 5.5 & $O(P I C)$ & 0.069 & 0.127 & 0.227 & 0.417 & 0.175 & 0.297 & 0.462 & 0.361 & 0.132 & 0.637 & 0.712 & 0.248 & 0.063 \\
\hline & & & 4.0 & 0.051 & 0.133 & 0.228 & 0.392 & 0.154 & 0.318 & 0.463 & 0.337 & 0.115 & 0.681 & 0.709 & 0.232 & 0.055 \\
\hline & & & 6.0 & 0.050 & 0.134 & 0.228 & 0.391 & 0.154 & 0.323 & 0.462 & 0.337 & 0.115 & 0.684 & 0.708 & 0.233 & 0.056 \\
\hline & & & 5.0 & 0.050 & 0.133 & 0.228 & 0.392 & 0.154 & 0.317 & 0.461 & 0.337 & 0.115 & 0.681 & 0.708 & 0.232 & 0.055 \\
\hline & 4.0 & 6.0 & $O(P I C)$ & 0.070 & 0.124 & 0.226 & 0.418 & 0.175 & 0.292 & 0.461 & 0.362 & 0.132 & 0.623 & 0.706 & 0.252 & 0.066 \\
\hline & & & 3.0 & 0.049 & 0.131 & 0.218 & 0.393 & 0.155 & 0.303 & 0.442 & 0.343 & 0.121 & 0.636 & 0.676 & 0.249 & 0.067 \\
\hline & & & 7.0 & 0.049 & 0.127 & 0.217 & 0.392 & 0.155 & 0.299 & 0.440 & 0.345 & 0.123 & 0.625 & 0.674 & 0.249 & 0.068 \\
\hline & & & 5.0 & 0.050 & 0.128 & 0.218 & 0.392 & 0.155 & 0.299 & 0.443 & 0.341 & 0.120 & 0.625 & 0.674 & 0.249 & 0.068 \\
\hline & 3.0 & 7.0 & $O(P I C)$ & 0.068 & 0.125 & 0.219 & 0.417 & 0.175 & 0.281 & 0.444 & 0.363 & 0.135 & 0.596 & 0.684 & 0.259 & 0.071 \\
\hline \multirow{12}{*}{ 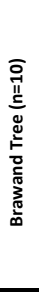 } & & & 4.5 & 0.050 & 0.159 & 0.234 & 0.337 & 0.114 & 0.416 & 0.476 & 0.284 & 0.081 & 0.823 & 0.725 & 0.188 & 0.036 \\
\hline & & & 5.5 & 0.050 & 0.159 & 0.236 & 0.336 & 0.113 & 0.419 & 0.476 & 0.284 & 0.082 & 0.822 & 0.725 & 0.188 & 0.036 \\
\hline & & & 5.0 & 0.050 & 0.162 & 0.234 & 0.338 & 0.114 & 0.418 & 0.474 & 0.285 & 0.082 & 0.825 & 0.726 & 0.186 & 0.035 \\
\hline & 4.5 & 5.5 & $O(P I C)$ & 0.074 & 0.150 & 0.234 & 0.367 & 0.135 & 0.368 & 0.471 & 0.311 & 0.098 & 0.758 & 0.722 & 0.207 & 0.044 \\
\hline & & & 4.0 & 0.049 & 0.157 & 0.233 & 0.337 & 0.114 & 0.414 & 0.472 & 0.288 & 0.084 & 0.813 & 0.720 & 0.190 & 0.037 \\
\hline & & & 6.0 & 0.050 & 0.159 & 0.234 & 0.336 & 0.113 & 0.411 & 0.471 & 0.286 & 0.082 & 0.813 & 0.719 & 0.190 & 0.037 \\
\hline & & & 5.0 & 0.049 & 0.161 & 0.233 & 0.337 & 0.114 & 0.413 & 0.470 & 0.286 & 0.083 & 0.819 & 0.721 & 0.188 & 0.036 \\
\hline & 4.0 & 6.0 & $O(P I C)$ & 0.074 & 0.148 & 0.233 & 0.368 & 0.136 & 0.366 & 0.471 & 0.310 & 0.097 & 0.752 & 0.719 & 0.209 & 0.045 \\
\hline & & & 3.0 & 0.050 & 0.158 & 0.229 & 0.338 & 0.115 & 0.399 & 0.461 & 0.289 & 0.085 & 0.789 & 0.702 & 0.196 & 0.041 \\
\hline & & & 7.0 & 0.049 & 0.150 & 0.224 & 0.339 & 0.115 & 0.388 & 0.456 & 0.292 & 0.087 & 0.774 & 0.696 & 0.201 & 0.043 \\
\hline & & & 5.0 & 0.049 & 0.156 & 0.227 & 0.335 & 0.113 & 0.398 & 0.458 & 0.288 & 0.085 & 0.790 & 0.699 & 0.197 & 0.041 \\
\hline & 3.0 & 7.0 & $O(P I C)$ & 0.071 & 0.147 & 0.228 & 0.364 & 0.133 & 0.366 & 0.465 & 0.310 & 0.097 & 0.746 & 0.710 & 0.209 & 0.045 \\
\hline \multirow{12}{*}{ 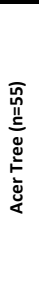 } & & & 4.5 & 0.049 & 0.576 & 0.247 & 0.129 & 0.017 & 0.989 & 0.496 & 0.105 & 0.011 & 1.000 & 0.745 & 0.062 & 0.004 \\
\hline & & & 5.5 & 0.049 & 0.578 & 0.247 & 0.129 & 0.017 & 0.989 & 0.495 & 0.104 & 0.011 & 1.000 & 0.744 & 0.062 & 0.004 \\
\hline & & & 5.0 & 0.050 & 0.577 & 0.248 & 0.129 & 0.017 & 0.989 & 0.495 & 0.104 & 0.011 & 1.000 & 0.745 & 0.062 & 0.004 \\
\hline & 4.5 & 5.5 & $O(P I C)$ & 0.080 & 0.503 & 0.247 & 0.144 & 0.021 & 0.971 & 0.495 & 0.117 & 0.014 & 1.000 & 0.745 & 0.070 & 0.005 \\
\hline & & & 4.0 & 0.051 & 0.566 & 0.245 & 0.130 & 0.017 & 0.988 & 0.491 & 0.105 & 0.011 & 1.000 & 0.739 & 0.063 & 0.004 \\
\hline & & & 6.0 & 0.050 & 0.570 & 0.245 & 0.129 & 0.017 & 0.988 & 0.491 & 0.105 & 0.011 & 1.000 & 0.738 & 0.064 & 0.004 \\
\hline & & & 5.0 & 0.049 & 0.572 & 0.245 & 0.129 & 0.017 & 0.988 & 0.491 & 0.105 & 0.011 & 1.000 & 0.739 & 0.063 & 0.004 \\
\hline & 4.0 & 6.0 & $O(P I C)$ & 0.081 & 0.493 & 0.245 & 0.144 & 0.021 & 0.969 & 0.492 & 0.117 & 0.014 & 1.000 & 0.742 & 0.070 & 0.005 \\
\hline & & & 3.0 & 0.049 & 0.552 & 0.239 & 0.130 & 0.017 & 0.984 & 0.478 & 0.106 & 0.012 & 1.000 & 0.718 & 0.066 & 0.005 \\
\hline & & & 7.0 & 0.050 & 0.546 & 0.235 & 0.130 & 0.017 & 0.982 & 0.471 & 0.107 & 0.012 & 1.000 & 0.709 & 0.069 & 0.007 \\
\hline & & & 5.0 & 0.048 & 0.552 & 0.237 & 0.129 & 0.017 & 0.984 & 0.475 & 0.106 & 0.012 & 1.000 & 0.713 & 0.067 & 0.006 \\
\hline & 3.0 & 7.0 & $O(P I C)$ & 0.075 & 0.494 & 0.241 & 0.143 & 0.021 & 0.968 & 0.483 & 0.117 & 0.014 & 1.000 & 0.727 & 0.072 & 0.006 \\
\hline
\end{tabular}


DisCUSSION

\section{Connection with Maximum Likelihood and GLS Method}

Similar to Felsenstein's PIC method, our OU-PIC method provides a tool for studying correlated evolution under the OU model. Our simulation results showed that appropriate type I error rates and the highest statistical power were achieved when the correct evolution model is specified. The statistical performance of contrast methods drops as the model assumptions deviate from the underlying evolution model. In this section, we discuss the connection between the results of the OU-PIC method and other methods in evaluating trait evolution.

\section{Connection with the Maximum Likelihood Estimation of the Optimal Trait Value}

Under the OU model of trait evolution, the vector of trait values $\boldsymbol{X}=\left(X_{1}, X_{2}, \cdots, X_{n}\right)^{T}$ follows a multivariate normal distribution, $\boldsymbol{X} \sim \mathcal{N}(\boldsymbol{\mu}, \Sigma)$. It is straight forward to find the maximum likelihood estimation of $\mu$ by taking derivatives of the log-likelihood,

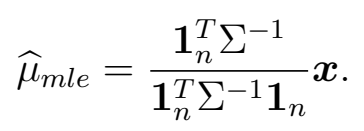

Here $\mathbf{1}_{n}$ is a column vector of $n$-ones. $\widehat{\mu}_{m l e}$ is an unbiased estimator of $\mu$.

$$
\mathbf{E}\left(\widehat{\mu}_{m l e}\right)=\mu \quad \text { and } \quad \operatorname{Var}\left(\widehat{\mu}_{m l e}\right)=\frac{1}{\mathbf{1}_{n}^{T \Sigma^{-1} \mathbf{1}_{n}} .}
$$

We found that the last intermediate value returned by our algorithm, $w_{n-1}$, is proportional to $\widehat{\mu}_{m l e}$, since their coefficients are linearly dependent (Appendix IV). Put in vector forms, we have

$$
\widehat{\mu}_{m l e}=\boldsymbol{a}^{T} \boldsymbol{x}, \quad w_{n-1}=\boldsymbol{b}^{T} \boldsymbol{x} \quad \text { and } \quad \boldsymbol{a} \| \boldsymbol{b} .
$$

In fact, both of them are independent to all $n-1$ contrasts (Appendix IV).

Connection with the GLS Method for Correlated Evolution In the GLS regression model, trait $Y$ is considered to be determined by trait $X$ plus some unspecified noise.

$$
Y=\beta_{0}+\beta_{1} X+\epsilon .
$$


The noise terms $\epsilon_{i}$ are assumed to be Gaussian with zero mean and covariance $\Sigma$, $\epsilon \sim N(0, \Sigma)$. Here, the structure of the covariance matrix describes the phylogenetic relationship among species, and is determined by the phylogenetic tree plus the evolutionary model (Hansen and Martins, 1996). Statistical tests were performed on the slop parameter $\beta_{1}$ in Equation (16) to test hypothesis about correlated evolution. It has long been noticed that when a BM model is assumed, the GLS estimation of the slope parameter in Equation (16), $\widehat{\beta}_{1}$, is equivalent to the slope parameter of the ordinary least squares regression of PICs conducted through the origin (Equation 17), $\widehat{\beta}_{p i c}$

$$
Y_{p i c}=\beta_{p i c} X_{p i c}+\epsilon_{p i c}
$$

Note that $\widehat{\beta}_{p i c}$ is different from the Pearson correlation coefficient $\left(r_{p i c}\right)$ of PICs, which is instead related to the slope parameter of the ordinary least squares regression between PICs with an intercept. Blomberg et al. (2012) provided a detailed proof of this equivalence using recursion, but as the authors pointed out, the proof involves some tedious algebraic derivations. We found that this equivalence still holds for our OU-PIC and the GLS regression method under the OU model provided that $\kappa_{x}=\kappa_{y}$.

We presented a convenient proof for this equivalence by analyzing the properties of the contrast coefficient matrix (Appendix V). In brief, we consider the vector of $n-1$ contrasts and $\widehat{\mu}_{m l e}$, with its coefficient matrix $G$.

$$
\boldsymbol{g}=\left[\begin{array}{c}
c_{1} \\
\vdots \\
c_{n-1} \\
\hat{\mu}_{m l e}
\end{array}\right] \quad G=\left[\begin{array}{cccc}
\mid & & \mid & \mid \\
\boldsymbol{p}_{\mathbf{1}}, & \cdots, & \boldsymbol{p}_{n-1}, & \boldsymbol{a} \\
\mid & & \mid & \mid
\end{array}\right]=\left[\begin{array}{ll}
P & \boldsymbol{a}
\end{array}\right] .
$$

Knowing that elements in $\boldsymbol{g}$ are normal distributed and independent to each other, $G$ is a linear transformation such that $G^{T} \Sigma G$ is diagonalized.

$$
\operatorname{Cov}(\boldsymbol{g})=G^{T} \Sigma G=\left[\begin{array}{cc}
\phi I_{n-1} & \mathbf{0} \\
\mathbf{0}^{T} & \frac{1}{\mathbf{1}_{n}^{T} \Sigma^{-1} \mathbf{1}_{n}}
\end{array}\right] .
$$

By substituting $G$ with $P$ and $\boldsymbol{a}$ in equation (19), we arrived at an identity between $\Sigma^{-1}$ and the coefficient matrix $P$. With that, it becomes straight forward to show that the two 
regression coefficients $\widehat{\beta}_{1}$ and $\widehat{\beta}_{\text {pic }}$ are equivalent.

As discussed previously (Blomberg et al., 2012), this equivalence suggests that ordinary regression analyses using PICs or OU-PICs have the same mathematical shortcomings as GLS methods. However, the algorithm for OU-PIC avoids the explicit calculation of the inverse of matrix $\Sigma$ and thus would be preferable when the species tree is large.

\section{Remarks on Method Limitations, Strengths, and Applications}

In this study, we investigated the evaluation of correlated evolution when the underlying evolutionary process is Ornstein-Uhlenbeck. By parameterizing the level of correlated evolution under the OU model, problems regarding correlated evolution were formulated to statistical tests or inferences on the correlated evolution parameter from correlated observations (species). Studies on statistical properties of methods for estimating correlation coefficients between two variables could be naturally applied here. Specifically, our OU-PIC algorithm provides a method to decorrelate samples and returns $n-1$ independent contrasts. We encountered theoretical difficulties when $\kappa_{x} \neq \kappa_{y}$, though this problem is not unique to the OU-PIC method. According to the regression model of the GLS method, trait $X$ is treated as the explanatory variable and trait $Y$ is considered as the dependent variable. When $\kappa_{x} \neq \kappa_{y}$, only the covariance structure of $Y$ is incorporated in the GLS regression model. However, we expect the covariance of the error terms to describe the covariance due to the stochastic evolution of both traits. For this reason, the GLS estimations are also subject to impacted statistical performance. To investigate this problem, we performed simulations to find that OU-PICs still achieve relatively good statistical performance when $\kappa_{x}$ and $\kappa_{y}$ diverges from each other but remains on the same scale.

Our results showed that the OU-PIC method is the most advantageous when moderate $\kappa$ is expected. If $\kappa \rightarrow 0$, the coefficients of OU-PICs get close to the coefficients 
of PICs. In this case, PICs also achieve good statistical performance in studying correlated evolution. On the contrary, if $\kappa$ is large $(\kappa>10$ for species trees we analyzed), the correlation among species resulted from overlapping evolutionary histories is negligible. The statistical performance of PIC may drop dramatically. Instead, raw observations from species could be directly supplied in statistical analyses. Our method has various applications when the trait evolution could be described by an OU process, for example the level of gene expressions that were frequently used in evolutionary studies recently (Rohlfs et al., 2014; Stewart et al., 2019; Kshitiz et al., 2019; Gu et al., 2019). Various studies have shown that the OU model is preferred in describing the evolution of gene expression in many clades (Bedford and Hartl, 2009; Metzger et al., 2017; Yang et al., 2017). Correlated evolution in gene expression has been widely observed among genes as well as between cell types (Ghanbarian and Hurst, 2015; Liang et al., 2018). Our OU-PIC method provides a convenient and statistically sound method to study correlated evolution under an OU process framework.

\section{(1) \\ CONCLUSions \\ In this study, we investigated correlated evolution under the OU model of trait evolution. We derived an extension of Felsenstein's PIC method under the OU model, called the OU-PIC method. We showed by simulation that our OU-PIC method improves the statistical performance of PIC in studying correlated evolution when stabilizing selection plays a non-negligible role. Our OU-PIC method avoids the calculation of the inverse of the covariance matrix and is straight forward to implement. It has many applications in evolutionary studies including molecular traits such as gene expressions.}

\section{Code Availability}

The OU-PIC method described in this manuscript will be available via a python package oupic on github. A developing version is also provided as supplementary material 
for review.

\section{ACKNOWLEDGEMENTS}

We thank Donghai Pan for helpful discussions. This work was supported by the National Natural Science Foundation of China grant no. 12001401.

\section{SupPlementary Material}

Supplementary figures and appendices are available from the Dryad Digital Repository: http://dx.doi.org/10.5061/dryad.xpnvx0kd4.

\section{REFERENCES}

D. D. Ackerly and M. J. Donoghue. Leaf size, sapling allometry, and Corner's rules: Phylogeny and correlated evolution in maples (Acer). American Naturalist, 152(6): 767-791, 1998. ISSN 00030147. doi: 10.1086/286208.

Trevor Bedford and Daniel L. Hartl. Optimization of gene expression by natural selection. Proceedings of the National Academy of Sciences of the United States of America, 106 (4):1133-1138, 2009. ISSN 00278424. doi: 10.1073/pnas.0812009106.

Simon P. Blomberg, James G. Lefevre, Jessie A. Wells, and Mary Waterhouse. Independent contrasts and PGLS regression estimators are equivalent. Systematic Biology, 61(3):382-391, 2012. ISSN 10635157. doi: 10.1093/sysbio/syr118.

Christine Böhmer, Olivia Plateau, Raphäel Cornette, and Anick Abourachid. Correlated evolution of neck length and leg length in birds. Royal Society Open Science, 6(5), 2019. ISSN 20545703. doi: 10.1098/rsos.181588.

David Brawand, Magali Soumillon, Anamaria Necsulea, Philippe Julien, Gábor Csárdi, Patrick Harrigan, Manuela Weier, Angélica Liechti, Ayinuer Aximu-Petri, Martin 
Kircher, Frank W. Albert, Ulrich Zeller, Philipp Khaitovich, Frank Grützner, Sven Bergmann, Rasmus Nielsen, Svante Pääbo, and Henrik Kaessmann. The evolution of gene expression levels in mammalian organs. Nature, 2011. ISSN 00280836. doi: 10.1038/nature10532.

Marguerite A. Butler and Aaron A. King. Phylogenetic comparative analysis: A modeling approach for adaptive evolution. American Naturalist, 164(6):683-695, 2004. ISSN 00030147. doi: $10.1086 / 426002$.

J. Felsenstein. Phylogenies and the comparative method. American Naturalist, 125(1): 1-15, 1985. ISSN 00030147. doi: 10.1086/284325.

Avazeh T. Ghanbarian and Laurence D. Hurst. Neighboring genes show correlated evolution in gene expression. Molecular Biology and Evolution, 32(7):1748-1766, 2015. ISSN 15371719. doi: 10.1093/molbev/msv053.

Xun Gu, Hang Ruan, and Jingwen Yang. Estimating the strength of expression conservation from high throughput RNA-seq data. Bioinformatics, 35(23):5030-5038, 2019. ISSN 14602059. doi: 10.1093/bioinformatics/btz405.

Thomas F. Hansen. Stabilizing Selection and the Comparative Analysis of Adaptation. Evolution, 51(5):1341, 1997. ISSN 00143820. doi: 10.2307/2411186.

Thomas F. Hansen and Emília P. Martins. Translating between microevolutionary process and macroevolutionary patterns: The correlation structure of interspecific data. Evolution, 50(4):1404-1417, 1996. ISSN 00143820. doi: 10.1111/j.1558-5646.1996.tb03914.x.

S. Blair Hedges, Julie Marin, Michael Suleski, Madeline Paymer, and Sudhir Kumar. Tree of life reveals clock-like speciation and diversification. Molecular Biology and Evolution, 32(4):835-845, 2015. ISSN 15371719. doi: 10.1093/molbev/msv037. 
REFERENCES

Lam Si Tung Ho and Cécile Ané. Asymptotic theory with hierarchical autocorrelation: Ornstein-Uhlenbeck tree models. Annals of Statistics, 41(2):957-981, 2013. ISSN 00905364. doi: 10.1214/13-AOS1105.

Lam Si Tung Ho and Cécile Ané. Intrinsic inference difficulties for trait evolution with Ornstein-Uhlenbeck models. Methods in Ecology and Evolution, 2014. ISSN 2041210X. doi: 10.1111/2041-210X.12285.

Mohammad Khabbazian, Ricardo Kriebel, Karl Rohe, and Cécile Ané. Fast and accurate detection of evolutionary shifts in Ornstein-Uhlenbeck models. Methods in Ecology and Evolution, 7(7):811-824, 2016. ISSN 2041210X. doi: 10.1111/2041-210X.12534.

Kshitiz, Junaid Afzal, Jamie D. Maziarz, Archer Hamidzadeh, Cong Liang, Eric M. Erkenbrack, Hong Nam, Jan Dirk Haeger, Christiane Pfarrer, Thomas Hoang, Troy Ott, Thomas Spencer, Mihaela Pavličev, Douglas F. Antczak, Andre Levchenko, and Günter P. Wagner. Evolution of placental invasion and cancer metastasis are causally linked. Nature Ecology and Evolution, 3(12):1743-1753, 2019. ISSN 2397334X. doi: 10.1038/s41559-019-1046-4. URL http://dx.doi.org/10.1038/s41559-019-1046-4.

Cong Liang, Jacob M. Musser, Alison Cloutier, Richard O. Prum, and Günter P. Wagner. Pervasive Correlated Evolution in Gene Expression Shapes Cell and Tissue Type Transcriptomes. Genome Biology and Evolution, 10(2):538-552, 2018. ISSN 17596653. doi: 10.1093/gbe/evy016.

Mark Pagel. Inferring historical patterns of biological evolution. Nature, 401(October 1999):877-884, 1999.

Emilia P. Martins and Theodore Garland. Phylogenetic Analyses of the Correlated Evolution of Continuous Characters: A Simulation Study. Evolution, 45(3):534, 1991. ISSN 00143820. doi: 10.2307/2409910.

Emília P. Martins and Thomas F. Hansen. Phylogenies and the comparative method: A 
general approach to incorporating phylogenetic information into the analysis of interspecific data. American Naturalist, 149(4):646-667, 1997. ISSN 00030147. doi: $10.1086 / 286013$.

Emilia P. Martins, Joseg Alexandre F. Diniz-Filho, and Elizabeth A. Housworth. Adaptive constraints and the phylogenetic comparative method: A computer simulation test. Evolution, 56(1):1-13, 2002. ISSN 00143820. doi: 10.1111/j.0014-3820.2002.tb00844.x.

Brian P.H. Metzger, Patricia J. Wittkopp, and Joseph D. Coolon. Evolutionary Dynamics of Regulatory Changes Underlying Gene Expression Divergence among Saccharomyces Species. Genome Biology and Evolution, 9(4):843-854, 2017. ISSN 17596653. doi: 10.1093/gbe/evx035.

J. Podos. Correlated evolution of morphology and vocal signal structure in Darwin's finches. Nature, 409(6817):185-188, 2001. ISSN 00280836. doi: 10.1038/35051570.

F. James Rohlf. a Comment on Phylogenetic Correction. Evolution, 60(7):1509, 2006. ISSN 0014-3820. doi: 10.1554/05-550.1.

Rori V. Rohlfs, Patrick Harrigan, and Rasmus Nielsen. Modeling gene expression evolution with an extended ornstein-uhlenbeck process accounting for within-species variation. Molecular Biology and Evolution, 31(1):201-211, 2014. ISSN 07374038. doi: $10.1093 / \mathrm{molbev} / \mathrm{mst} 190$.

Thomas A. Stewart, Cong Liang, Justin L. Cotney, James P. Noonan, Thomas J. Sanger, and Günter P. Wagner. Evidence against tetrapod-wide digit identities and for a limited frame shift in bird wings. Nature Communications, 10(1), 2019. ISSN 20411723. doi: 10.1038/s41467-019-11215-8. URL http://dx.doi.org/10.1038/s41467-019-11215-8.

Jian-rong Yang, Calum J Maclean, Chungoo Park, Huabin Zhao, and Jianzhi Zhang. Intra and Interspecific Variations of Gene Expression Levels in Yeast Are Largely Neutral : ( 
Nei Lecture, SMBE 2016 , Gold Coast ) Article Fast Track. 34(9):2125-2139, 2017. doi: $10.1093 / \mathrm{molbev} / \mathrm{msx} 171$.

Jingwen Yang, Hang Ruan, Wenjie Xu, and Xun Gu. TreeExp2: An Integrated Framework for Phylogenetic Transcriptome Analysis. Genome biology and evolution, 11(11): 3276-3282, 2019. ISSN 17596653. doi: 10.1093/gbe/evz222. 
We show that under the OU model with the stationary assumption, the covariance of trait $X$ between nodes $i$ and $j$ is:

$$
\operatorname{Cov}\left(X_{i}, X_{j}\right)=\frac{\sigma^{2}}{2 \lambda} e^{-\lambda d(i, j)}
$$

Proof. Let $t_{i}, t_{j}$ be the evolution time between $i, j$ and their last common ancestor $k$, respectively. Consider the evolutionary process from $k(t=0)$ to $i\left(t=t_{i}\right)$,

$$
X_{i}=\mu+\left(X_{k}-\mu\right) e^{-\lambda t_{i}}+\sigma \int_{0}^{t_{i}} e^{-\lambda\left(t_{i}-s\right)} d W_{s}^{(i)}
$$

and the process from $k(t=0)$ to $j\left(t=t_{j}\right)$,

$$
X_{j}=\mu+\left(X_{k}-\mu\right) e^{-\lambda t_{j}}+\sigma \int_{0}^{t_{j}} e^{-\lambda\left(t_{j}-s\right)} d W_{s}^{(j)}
$$

Subscripts for $\mu$ and $\lambda$ are omitted here for the simplicity of the notation. With the stationary assumption, $X_{i}, X_{j}, X_{k} \sim N\left(\mu, \frac{\sigma^{2}}{2 \lambda}\right)$. The evolutionary processes are considered independent after the speciation event, i.e. $\operatorname{Cov}\left(d W_{s}^{(i)}, d W_{s}^{(j)}\right)=0$. We have,

$$
\begin{aligned}
\operatorname{Cov}\left(X_{i}, X_{j}\right)= & \mathbf{E}\left(X_{i}-\mu\right)\left(X_{j}-\mu\right) \\
= & \mathbf{E}\left(\left(X_{k}-\mu\right) e^{-\lambda t_{i}}+\sigma \int_{0}^{t_{i}} e^{-\lambda\left(t_{i}-s\right)} d W_{s}^{(i)}\right)\left(\left(X_{k}-\mu\right) e^{-\lambda t_{j}}+\sigma \int_{0}^{t_{j}} e^{-\lambda\left(t_{j}-s\right)} d W_{s}^{(j)}\right) \\
= & \mathbf{E}\left(\left(X_{k}-\mu\right)^{2} e^{-\lambda\left(t_{i}+t_{j}\right)}\right)+\mathbf{E}\left(\left(X_{k}-\mu\right) e^{-\lambda t_{i}}\right)\left(\sigma \int_{0}^{t_{j}} e^{-\lambda\left(t_{j}-s\right)} d W_{s}^{(j)}\right) \\
& +\mathbf{E}\left(\left(X_{k}-\mu\right) e^{-\lambda t_{j}}\right)\left(\sigma \int_{0}^{t_{i}} e^{-\lambda\left(t_{i}-s\right)} d W_{s}^{(i)}\right) \\
& +\sigma^{2} \mathbf{E}\left(\int_{0}^{t_{i}} e^{-\lambda\left(t_{i}-s\right)} d W_{s}^{(i)}\right)\left(\int_{0}^{t_{j}} e^{-\lambda\left(t_{j}-s\right)} d W_{s}^{(j)}\right) \\
= & \mathbf{E}\left(X_{k}-\mu\right)^{2} e^{-\lambda\left(t_{i}+t_{j}\right)} \\
= & \frac{\sigma^{2}}{2 \lambda} e^{-\lambda d(i, j)}
\end{aligned}
$$


We show that under the OU model with the stationary assumption, the covariance between two traits $X$ and $Y$ on node $k$ is

$$
\operatorname{Cov}\left(X_{k}, Y_{k}\right)=\gamma_{x y} \cdot \frac{\sigma_{x} \sigma_{y}}{\left(\lambda_{x}+\lambda_{y}\right)}
$$

Proof. Consider the the evolutionary process from an ancient ancestor $\alpha(t=0)$ to node $k$ $\left(t=\tau_{k}\right)$. Here $\alpha$ is much more ancient than the last common ancestor of the species in the given phylogeny. We have,

$$
X_{k}=\mu_{x}+\left(x_{\alpha}-\mu_{x}\right) e^{-\lambda_{x} \tau_{k}}+\sigma_{x} \int_{0}^{\tau_{k}} e^{-\lambda_{x}\left(\tau_{k}-s\right)} d W_{s}
$$

and

$$
Y_{k}=\mu_{y}+\left(y_{\alpha}-\mu_{y}\right) e^{-\lambda_{y} \tau_{k}}+\sigma_{y} \int_{0}^{\tau_{k}} e^{-\lambda_{y}\left(\tau_{k}-s\right)} d W_{s}^{\prime}
$$

With direct calculation,

$$
\begin{aligned}
\operatorname{Cov}\left(X_{k}, Y_{k}\right) & =\mathbf{E}\left(X_{k}-\mathbf{E} X_{k}\right)\left(Y_{k}-\mathbf{E} Y_{k}\right) \\
& =\mathbf{E}\left(\int_{0}^{\tau_{k}} \sigma_{x} e^{-\lambda_{x}\left(\tau_{k}-u\right)} d W_{u}\right)\left(\int_{0}^{\tau_{k}} \sigma_{y} e^{-\lambda_{y}\left(\tau_{k}-v\right)} d W_{v}^{\prime}\right) \\
& =\gamma_{x y} \cdot \sigma_{x} \sigma_{y} \cdot e^{-\left(\lambda_{x}+\lambda_{y}\right) \tau_{k}} \cdot \mathbf{E}\left(\int_{0}^{\tau_{k}} e^{\lambda_{x} u} d W_{u}\right)\left(\int_{0}^{\tau_{k}} e^{\lambda_{y} v} d W_{v}\right) \\
& =\gamma_{x y} \cdot \sigma_{x} \sigma_{y} \cdot e^{-\left(\lambda_{x}+\lambda_{y}\right) \tau_{k}} \cdot\left(\int_{0}^{\tau_{k}} e^{\left(\lambda_{x}+\lambda_{y}\right) u} d u\right) \\
& =\gamma_{x y} \cdot \frac{\sigma_{x} \sigma_{y}}{\left(\lambda_{x}+\lambda_{y}\right)} \cdot\left(1-e^{-\left(\lambda_{x}+\lambda_{y}\right) \tau_{k}}\right)
\end{aligned}
$$

The stationary assumption suggests that the time from the ancient ancestor to the root of phylogeny is long enough and thus can be considered as $\tau_{k} \rightarrow \infty$.

$$
\begin{aligned}
\operatorname{Cov}\left(X_{k}, Y_{k}\right) & =\gamma_{x y} \cdot \frac{\sigma_{x} \sigma_{y}}{\left(\lambda_{x}+\lambda_{y}\right)} \cdot \lim _{\tau_{k} \rightarrow \infty}\left(1-e^{-\left(\lambda_{x}+\lambda_{y}\right) \tau_{k}}\right) \\
& =\gamma_{x y} \cdot \frac{\sigma_{x} \sigma_{y}}{\left(\lambda_{x}+\lambda_{y}\right)}
\end{aligned}
$$

We show that under the OU model with the stationary assumption, the covariance 
between trait $X$ on node $i$ and trait $Y$ on node $j$ is:

$$
\operatorname{Cov}\left(X_{i}, Y_{j}\right)=\gamma_{x y} \cdot \frac{\sigma_{x} \sigma_{y}}{\left(\lambda_{x}+\lambda_{y}\right)} \cdot e^{-\left(\lambda_{x} t_{i}+\lambda_{y} t_{j}\right)}
$$

With $t_{i}, t_{j}$ as the evolution time between $i, j$ and their last common ancestor $k$,

respectively.

Proof. Consider the evolutionary process from $k(t=0)$ to $i\left(t=t_{i}\right)$ and the process from $k(t=0)$ to $j\left(t=t_{j}\right)$. With the stationary assumption, $X_{i}, X_{j}, X_{k} \sim N\left(\mu, \frac{\sigma^{2}}{2 \lambda}\right)$. We have,

$$
\begin{aligned}
& \operatorname{Cov}\left(X_{i}, Y_{j}\right) \\
= & \mathbf{E}\left(X_{i}-\mu_{x}\right)\left(Y_{j}-\mu_{y}\right) \\
= & \mathbf{E}\left(\left(X_{k}-\mu_{x}\right) e^{-\lambda_{x} t_{i}}+\sigma_{x} \int_{0}^{t_{i}} e^{-\lambda_{x}\left(t_{i}-s\right)} d W_{s}\right)\left(\left(Y_{k}-\mu_{y}\right) e^{-\lambda_{y} t_{j}}+\sigma_{y} \int_{0}^{t_{j}} e^{-\lambda_{y}\left(t_{j}-s\right)} d W_{s}^{\prime}\right) \\
= & \mathbf{E}\left(X_{k}-\mu_{x}\right)\left(Y_{k}-\mu_{y}\right) e^{-\left(\lambda_{x} t_{i}+\lambda_{y} t_{j}\right)}+\sigma_{y} \cdot \mathbf{E}\left(\left(X_{k}-\mu_{x}\right) e^{-\lambda_{x} t_{i}}\right)\left(\int_{0}^{t_{j}} e^{-\lambda_{y}\left(t_{j}-s\right)} d W_{s}\right) \\
& +\sigma_{x} \cdot \mathbf{E}\left(\left(Y_{k}-\mu_{y}\right) e^{-\lambda_{y} t_{j}}\right)\left(\int_{0}^{t_{i}} e^{-\lambda_{x}\left(t_{i}-s\right)} d W_{s}^{\prime}\right) \\
& +\sigma_{x} \cdot \sigma_{y} \cdot \mathbf{E}\left(\int_{0}^{t_{i}} e^{-\lambda_{x}\left(t_{i}-s\right)} d W_{s}\right)\left(\int_{0}^{t_{j}} e^{-\lambda_{y}\left(t_{j}-s\right)} d W_{s}^{\prime}\right) \\
= & \mathbf{E}\left(X_{k}-\mu_{x}\right)\left(Y_{k}-\mu_{y}\right) e^{-\left(\lambda_{x} t_{i}+\lambda_{y} t_{j}\right)} \\
= & \operatorname{Cov}\left(X_{k}, Y_{k}\right) \cdot e^{-\left(\lambda_{x} t_{i}+\lambda_{y} t_{j}\right)} \\
= & \gamma_{x y} \cdot \frac{\sigma_{x} \sigma_{y}}{\lambda_{x}+\lambda_{y}} \cdot e^{-\left(\lambda_{x} t_{i}+\lambda_{y} t_{j}\right)}
\end{aligned}
$$

The coefficients for calculating contrasts and intermediate values in the OU-PIC algorithm were given by the following equations. 


$$
\begin{aligned}
& \eta_{i}=\sqrt{\frac{2}{e^{-2 \lambda v_{r}}+e^{-2 \lambda v_{l}}-2 e^{-2 \lambda\left(v_{r}+v_{l}\right)}}} ; \\
& a_{i}=t\left(e^{\lambda v_{r}}-e^{-\lambda v_{r}}\right), \quad b_{i}=t\left(e^{\lambda v_{l}}-e^{-\lambda v_{l}}\right) ; \\
& t=\frac{1}{\sqrt{\left(e^{2 \lambda v_{l}}+e^{2 \lambda v_{r}}-2\right)\left(1-e^{-2 \lambda\left(v_{l}+v_{r}\right)}\right)}} ; \\
& \Delta d\left(\mathbf{w}_{i}, \mathbf{w}_{i}^{a n c}\right)=\frac{1}{\lambda} \log \sqrt{\frac{e^{2 \lambda\left(v_{r}+v_{l}\right)}-1}{e^{2 \lambda v_{l}}+e^{2 \lambda v_{r}}-2}} .
\end{aligned}
$$

returned by the OU-PIC algorithm have the following properties:

1. $C_{i} \sim N(0, \phi), \phi$ is the normalized variance for all contrasts;

2. $C_{i}$ are independent to each other;

3. if two traits $X$ and $Y$ have the same level of stabilizing selection, the Pearson correlation coefficient between their contrasts is $\gamma_{x y}$ :

$$
\operatorname{Corr}\left(C_{i}^{X}, C_{j}^{Y}\right)= \begin{cases}\gamma_{x y}, & \text { if } i=j \\ 0, & \text { if } i \neq j\end{cases}
$$

Proof. We refer to the phylogeny at the beginning of $i$-th iteration as the $i$-th tree, and denote the divergence time between nodes $\mathrm{a}$ and $\mathrm{b}$ on the $i$-th tree as $d^{i}(\mathrm{a}, \mathrm{b})$. In $i$-th iteration, two leaf nodes $\mathrm{x}_{l}$ and $\mathrm{x}_{r}$ were trimmed from the $i$-th tree, and their last common ancestor $\mathrm{w}_{\mathrm{i}}$ becomes a leaf node in the $(i+1)$-th tree.

(1) Since $C_{i}$ 's are linear combinations of trait values on leaf nodes, $C_{i}$ 's are also normal distributed. According to the algorithm, variances of $C_{i}$ 's are normalized to $\phi=\frac{\sigma^{2}}{\lambda}$. It only remains to show that $\mathbf{E} C_{i}=0$. We show by recursion that $\frac{\mathbf{E} X_{k}}{e^{-\lambda d^{i}(\text { root }, k)}}$ remains a constant for all leaf nodes $k$ in all iterations.

(i) When $i=1$, since the initial species tree has only extant species, the evolution time from the root to any extant species is the same. Denote $d^{1}($ root,$k)=L$. With the stationary assumption, $\mathbf{E} X_{k}=\mu$. Thus,

$$
\frac{\mathbf{E} X_{k}}{e^{-\lambda d^{1}(r o o t, k)}}=\frac{\mu}{e^{-\lambda L}}=\frac{\mu}{D}
$$


(ii) In $i$-th iteration, $\mathrm{w}_{i}$ becomes a new leaf node with modified branch length, while other nodes remain unchanged. To show that the tree pruning procedure introduced in our algorithm does not change the above constant, we only need to verify that the above ratio for node $w_{i}$ remains the same in the $(i+1)$-th tree. The numerator,

$$
\mathbf{E} W_{i}=a_{i} \mathbf{E} X_{l}+b_{i} \mathbf{E} X_{r}
$$

The denominator,

$$
\begin{aligned}
e^{\left.-\lambda d^{i+1}\left(\text { root }, \mathrm{w}_{i}\right)\right)} & =e^{-\lambda\left(d^{i}\left(\text { root }, \mathrm{w}_{i}\right),+\Delta d\left(\mathrm{w}_{i}^{a s}, \mathrm{w}_{i}\right)\right)} \\
& =e^{-\lambda\left(d^{i}\left(\text { root }, \mathrm{w}_{i}\right)\right)}\left(a_{i} e^{-\lambda v_{l}}+b_{i} e^{-\lambda v_{r}}\right) \\
& =a_{i} e^{-\lambda d^{i}\left(\text { root }, \mathrm{x}_{l}\right)}+b_{i} e^{-\lambda d^{i}\left(\text { root }, \mathrm{x}_{r}\right)}
\end{aligned}
$$

Since $\frac{\mathbf{E} X_{l}}{e^{-\lambda d^{i}\left({\left.\text { root }, \mathrm{x}_{l}\right)}_{1}\right.}}=\frac{\mathbf{E} X_{r}}{e^{-\lambda d^{i}\left({\left.\text { root }, \mathrm{x}_{r}\right)}\right.}}=\frac{\mu}{D}$, we have,

$$
\frac{\mathbf{E} W_{i}}{e^{-\lambda d^{i+1}\left(\text { root }, \mathrm{w}_{i}\right)}}=\frac{a_{i} \mathbf{E} X_{l}+b_{i} \mathbf{E} X_{r}}{a_{i} e^{-\lambda d^{i}\left(r o o t, x_{l}\right)}+b_{i} e^{-\lambda d^{i}\left(\text { root }, \mathrm{x}_{r}\right)}}=\frac{\mu}{D} .
$$

532 As a result, if $\frac{\mathbf{E} X_{k}}{e^{-\lambda d^{i}(\text { root }, k)}}=\frac{\mu}{D}$ is true for any leaf node $k$ in the $i$-th tree, then ${ }_{533} \frac{\mathbf{E} X_{k}}{e^{-\lambda d^{i+1}(\text { root }, k)}}=\frac{\mu}{D}$ also holds for all leaf nodes in the $(i+1)$-th tree.

With (i) and (ii), $\frac{\mathbf{E} X_{k}}{e^{-\lambda d^{i}(\text { root }, k)}}=\frac{\mu}{D}$ is a constant for all leaf nodes in all iterations. We find the expectation of the $i$-th contrast,

$$
\begin{aligned}
\mathbf{E} C_{i} & =\eta_{i} e^{-\lambda d^{i}\left(\mathrm{w}_{i}, \mathrm{x}_{r}\right)} \mathbf{E} X_{l}-\eta_{i} e^{-\lambda d^{i}\left(\mathrm{w}_{i}, \mathrm{x}_{l}\right)} \mathbf{E} X_{r} \\
& =\eta_{i} \frac{\mu}{D}\left(e^{-\lambda\left(d^{i}\left(\mathrm{w}_{i}, \mathrm{x}_{r}\right)+d^{i}\left(\text { root }, \mathrm{x}_{l}\right)\right)}-e^{-\lambda\left(d^{i}\left(\mathrm{w}_{i}, \mathrm{x}_{l}\right)+d^{i}\left(\text { root }, \mathrm{x}_{r}\right)\right)}\right) \\
& =\eta_{i} \frac{\mu}{D}\left(e^{-\lambda\left(d^{i}\left(\mathrm{w}_{i}, \mathrm{x}_{r}\right)+d^{i}\left(\mathrm{w}_{i}, \mathrm{x}_{l}\right)+d^{i}\left(\text { root }, \mathrm{w}_{i}\right)\right)}-e^{-\lambda\left(d^{i}\left(\mathrm{w}_{i}, \mathrm{x}_{l}\right)+d^{i}\left(\mathrm{w}_{i}, \mathrm{x}_{r}\right)+d^{i}\left(\text { root }, \mathrm{w}_{i}\right)\right)}\right) \\
& =0
\end{aligned}
$$

(2) We first find the covariance between node $w_{i}$ and any other unmodified leaf node $k$ in $i$-th iteration: 


$$
\begin{aligned}
\operatorname{Cov}\left(W_{i}, X_{k}\right) & =\operatorname{Cov}\left(a_{i} X_{l}+b_{i} X_{r}, X_{k}\right) \\
& =a_{i} \operatorname{Cov}\left(X_{l}, X_{k}\right)+b_{i} \operatorname{Cov}\left(X_{r}, X_{k}\right) \\
& =\frac{\sigma^{2}}{2 \lambda}\left(a_{i} e^{-\lambda d^{i}\left(\mathrm{x}_{l}, k\right)}+b_{i} e^{-\lambda d^{i}\left(\mathrm{x}_{r}, k\right)}\right) \\
& =\frac{\sigma^{2}}{2 \lambda}\left(a_{i} e^{-\lambda\left(d^{i}\left(\mathrm{x}_{l}, \mathrm{w}_{i}\right)+d^{i}\left(\mathrm{w}_{i}, k\right)\right)}+b_{i} e^{-\lambda\left(d^{i}\left(\mathrm{x}_{r}, \mathrm{w}_{i}\right)+d^{i}\left(\mathrm{w}_{i}, k\right)\right)}\right) \\
& =\frac{\sigma^{2}}{2 \lambda} e^{-\lambda d^{i}\left(\mathrm{w}_{i}, k\right)}\left(a_{i} e^{-\lambda v_{l}}+b_{i} e^{-\lambda v_{r}}\right) \\
& =\frac{\sigma^{2}}{2 \lambda} e^{-\lambda\left(d^{i}\left(\mathrm{w}_{i}, k\right)+\Delta d\left(W_{i}^{a s}, \mathrm{w}_{i}\right)\right)} \\
& =\frac{\sigma^{2}}{2 \lambda} e^{-\lambda d^{i+1}\left(\mathrm{w}_{i}, k\right)}
\end{aligned}
$$

${ }_{536}$ Knowing that $\operatorname{Var}\left(W_{i}\right)=\frac{\sigma^{2}}{2 \lambda}$, equation (20) about the covariances among leaf nodes still holds in the $(i+1)$-th tree.

Next, we show that the covariance between the contrast $C_{i}$ and any leaf in the $(i+1)$-th tree (the pruned tree) is zero. According to the algorithm, $\operatorname{Cov}\left(C_{i}, W_{i}\right)=0$. For other unmodified leaf nodes,

$$
\begin{aligned}
\operatorname{Cov}\left(C_{i}, X_{k}\right) & =\operatorname{Cov}\left(\eta_{i} e^{-\lambda v_{r}} X_{l}-\eta_{i} e^{-\lambda v_{l}} X_{r}, X_{k}\right) \\
& =\eta_{i} e^{-\lambda v_{r}} \operatorname{Cov}\left(X_{l}, X_{k}\right)-\eta_{i} e^{-\lambda v_{l}} \operatorname{Cov}\left(X_{r}, X_{k}\right) \\
& =\frac{\sigma^{2}}{2 \lambda} \eta_{i}\left(e^{-\lambda\left(v_{r}+d^{i}\left(\mathrm{x}_{l}, k\right)\right)}-e^{-\lambda\left(v_{l}+d^{i}\left(\mathrm{x}_{r}, k\right)\right)}\right) \\
& =\frac{\sigma^{2}}{2 \lambda} \eta_{i}\left(e^{-\lambda\left(v_{r}+v_{l}+d^{i}\left(\mathrm{w}_{i}, k\right)\right)}-e^{-\lambda\left(v_{l}+v_{r}+d^{i}\left(\mathrm{w}_{i}, k\right)\right)}\right) \\
& =0
\end{aligned}
$$

Since any $C_{j}$ with $j>i$ is some linear combination of the leaf node values in the $i$-th tree, we have $\operatorname{Cov}\left(C_{j}, C_{i}\right)=0$ for $j \neq i$. As a result, $C_{i}$ are independent to each other.

(3) Consider two traits $X$ and $Y$ with $\lambda_{x}=\lambda_{y}=\lambda$. According to Equation (23), they obtain the same coefficients for contrasts, intermediate values and branch lengths. First, we show by recursion that equations (21) and (22) hold for all trees when 
$\lambda_{x}=\lambda_{y}$, i.e.

$$
\operatorname{Cov}\left(X_{k}, Y_{k}\right)=\gamma_{x y} \cdot \frac{\sigma_{x} \sigma_{y}}{2 \lambda}, \quad \operatorname{Cov}\left(X_{i}, Y_{j}\right)=\gamma_{x y} \cdot \frac{\sigma_{x} \sigma_{y}}{2 \lambda} \cdot e^{-\lambda d^{i+1}(i, j)}
$$

When $i=1$, we know that equations (24) are true for the original species tree. If equations (24) hold in the $i$-th tree, it is straight forward to verify that the covariance between the values on node $\mathrm{w}_{i}, \operatorname{Cov}\left(W_{i}^{X}, W_{i}^{Y}\right)=\gamma_{x y} \cdot \frac{\sigma_{x} \sigma_{y}}{2 \lambda}$, and that the covariance between trait $X$ on node $\mathrm{w}_{i}$ and trait $Y$ on any unmodified node $k, \operatorname{Cov}\left(W_{i}^{X}, Y_{k}\right)=\gamma_{x y} \cdot \frac{\sigma_{x} \sigma_{y}}{2 \lambda} \cdot e^{-\lambda d^{i+1}\left(\mathrm{w}_{i}, k\right)}$. Since the rest of the nodes and branch lengths remains unchanged in $i$-th iteration, equations $(24)$ holds for $(i+1)$-th tree. As a result, equations $(24)$ are true for trees in all iterations.

Next, we directly verify that $\operatorname{Corr}\left(C_{i}^{X}, C_{i}^{Y}\right)=\gamma_{x y}$.

$$
\begin{aligned}
\operatorname{Cov}\left(C_{i}^{X}, C_{i}^{Y}\right)= & \operatorname{Cov}\left(\eta_{i} e^{-\lambda v_{r}} X_{l}-\eta_{i} e^{-\lambda v_{l}} X_{r}, \eta_{i} e^{-\lambda v_{r}} Y_{l}-\eta_{i} e^{-\lambda v_{l}} Y_{r}\right) \\
= & \eta_{i}^{2} e^{-2 \lambda v_{r}} \operatorname{Cov}\left(X_{l}, Y_{l}\right)-\eta_{i}^{2} e^{-\lambda\left(v_{l}+v_{r}\right)} \operatorname{Cov}\left(X_{r}, Y_{l}\right) \\
& -\eta_{i}^{2} e^{-\lambda\left(v_{r}+v_{l}\right)} \operatorname{Cov}\left(X_{l}, Y_{r}\right)+\eta_{i}^{2} e^{-2 \lambda v_{l}} \operatorname{Cov}\left(X_{r}, Y_{r}\right) \\
= & \eta_{i}^{2}\left(e^{-2 \lambda v_{r}}+e^{-2 \lambda v_{l}}-2 e^{-2 \lambda\left(v_{l}+v_{r}\right)}\right) \gamma_{x y} \frac{\sigma_{x} \sigma_{y}}{\lambda} \\
= & \gamma_{x y} \frac{\sigma_{x} \sigma_{y}}{\lambda}
\end{aligned}
$$

Knowing that $\operatorname{Var}\left(C_{i}^{X}\right)=\frac{\sigma_{x}^{2}}{\lambda}$ and that $\operatorname{Var}\left(C_{i}^{Y}\right)=\frac{\sigma_{y}^{2}}{\lambda}$, we have

$$
\operatorname{Corr}\left(C_{i}^{X}, C_{i}^{Y}\right)=\frac{\operatorname{Cov}\left(C_{i}^{X}, C_{i}^{Y}\right)}{\sqrt{\operatorname{Var}\left(C_{i}^{X}\right) \operatorname{Var}\left(C_{i}^{Y}\right)}}=\gamma_{x y}
$$

Finally, we show that $\operatorname{Cov}\left(C_{i}^{X}, C_{j}^{Y}\right)=0,(i \neq j)$. It is sufficient to show this is true when $j>i$. When $j>i, C_{j}^{Y}$ is a linear combination of $Y$ values on the $(i+1)$-th tree. It is sufficient to show that (i) the covariance between $C_{i}^{X}$ and $W_{i}^{Y}$ is zero, and that (ii) the covariance between $C_{i}^{X}$ and trait $Y$ on other unmodified leaf nodes in $i$-th iteration are all zero. 
(i) We directly verify the covariance between $C_{i}^{X}$ and $W_{i}^{Y}$.

$$
\begin{aligned}
\operatorname{Cov}\left(C_{i}^{X}, W_{i}^{Y}\right)= & \operatorname{Cov}\left(\eta_{i} e^{-\lambda v_{r}} X_{l}-\eta_{i} e^{-\lambda v_{l}} X_{r}, a_{i} Y_{l}+b_{i} Y_{r}\right) \\
\propto & e^{-\lambda v_{r}} a_{i} \operatorname{Cov}\left(X_{l}, Y_{l}\right)-e^{-\lambda v_{l}} a_{i} \operatorname{Cov}\left(X_{r}, Y_{l}\right) \\
& +e^{-\lambda v_{r}} b_{i} \operatorname{Cov}\left(X_{l}, Y_{r}\right)-e^{-\lambda v_{l}} b_{i} \operatorname{Cov}\left(X_{r}, Y_{r}\right) \\
\propto & e^{-\lambda v_{r}}\left(e^{\lambda v_{r}}-e^{-\lambda v_{r}}\right)-e^{-\lambda v_{l}}\left(e^{\lambda v_{r}}-e^{-\lambda v_{r}}\right) e^{-\lambda\left(v_{r}+v_{l}\right)} \\
& +e^{-\lambda v_{r}}\left(e^{\lambda v_{l}}-e^{-\lambda v_{l}}\right) e^{-\lambda\left(v_{r}+v_{l}\right)}-e^{-\lambda v_{l}}\left(e^{\lambda v_{l}}-e^{-\lambda v_{l}}\right) \\
= & 0
\end{aligned}
$$

(ii) Denote the last common ancestor of node $\mathrm{w}_{\mathrm{i}}$ and node $k$ as node $\mathrm{kw} . k$ is a leaf node on $i$-th tree which was not modified in $i$-th iteration.

$$
\begin{aligned}
\operatorname{Cov}\left(C_{i}^{X}, Y_{k}\right) & =\operatorname{Cov}\left(\eta_{i} e^{-\lambda v_{r}} X_{l}-\eta_{i} e^{-\lambda v_{l}} X_{r}, Y_{k}\right) \\
& =\eta_{i}^{2} e^{-\lambda v_{r}} \operatorname{Cov}\left(X_{l}, Y_{k}\right)-\eta_{i}^{2} e^{-\lambda v_{l}} \operatorname{Cov}\left(X_{r}, Y_{k}\right) \\
& \propto e^{-\lambda\left(v_{r}+v_{l}+d^{i}\left(\mathrm{kw}, \mathrm{w}_{\mathrm{i}}\right)+d^{i}(\mathrm{kw}, k)\right)}-e^{-\lambda\left(v_{r}+v_{l}+d^{i}\left(\mathrm{kw}, \mathrm{w}_{\mathrm{i}}\right)+d^{i}(\mathrm{kw}, k)\right)} \\
& =0
\end{aligned}
$$
maximum likelihood estimation (MLE) of the optimal value $\widehat{\mu}_{m l e}$.

Proof. We first find $\widehat{\mu}_{m l e}$, the maximum likelihood estimator of $\mu_{x}$. The vector of trait values $\boldsymbol{X}=\left(X_{1}, X_{2}, \cdots, X_{n}\right)^{T}$ follows a multivariate normal distribution, $\boldsymbol{X} \sim \mathcal{N}(\boldsymbol{\mu}, \Sigma)$. Here, $\boldsymbol{\mu}=(\mu, \cdots, \mu)^{T}=\mathbf{1}_{n} \mu$. We denote $\mathbf{1}_{n}=(1, \cdots, 1)^{T}$, and $\mathbf{0}_{n}=(0, \cdots, 0)^{T}$.

The probability density of $\boldsymbol{X}$ is

$$
\mathbf{P}(\boldsymbol{X}=\boldsymbol{x} \mid \mu)=(2 \pi)^{n / 2} \operatorname{det}(\Sigma)^{-1 / 2} \exp \left(-\frac{1}{2}(\boldsymbol{x}-\boldsymbol{\mu})^{T} \Sigma^{-1}(\boldsymbol{x}-\boldsymbol{\mu})\right)
$$


The log-likelihood

$$
\mathcal{L}(\mu)=\frac{n}{2} \log (2 \pi)-\frac{1}{2} \log [\operatorname{det}(\Sigma)]-\frac{1}{2}\left(\boldsymbol{x}-\mathbf{1}_{n} \mu\right)^{T} \Sigma^{-1}\left(\boldsymbol{x}-\mathbf{1}_{n} \mu\right) .
$$

${ }_{563}$ Since $\frac{\partial^{2} \mathcal{L}}{\partial \mu^{2}}=-\mathbf{1}_{n}^{T} \Sigma^{-1} \mathbf{1}_{n}<0$. Let $\frac{\partial \mathcal{L}}{\partial \mu}=0$, we find the MLE of $\mu$,

$$
\widehat{\mu}_{m l e}=\frac{\mathbf{1}_{n}^{T} \Sigma^{-1}}{\mathbf{1}_{n}^{T} \Sigma^{-1} \mathbf{1}_{n}} \boldsymbol{x} .
$$

With

$$
\mathbf{E}\left(\widehat{\mu}_{m l e}\right)=\mu, \quad \operatorname{Var}\left(\widehat{\mu}_{m l e}\right)=\frac{1}{\mathbf{1}_{n}^{T \Sigma^{-1} \mathbf{1}_{n}} .}
$$

564

Recall their vector and matrix notations, $\widehat{\mu}_{m l e}=\boldsymbol{a}^{T} \boldsymbol{x}, w_{n-1}=\boldsymbol{b}^{T} \boldsymbol{x}, c_{i}=\boldsymbol{p}_{\boldsymbol{i}}^{T} \boldsymbol{x}$ and $P=\left(\boldsymbol{p}_{\mathbf{1}}, \boldsymbol{p}_{\mathbf{2}}, \cdots, \boldsymbol{p}_{\boldsymbol{n}-\mathbf{1}}\right)$. We next show that $\boldsymbol{a}$ and $\boldsymbol{b}$ is linearly dependent to each other $(\boldsymbol{a} \| \boldsymbol{b})$.

From equation (25), we have $\boldsymbol{a}^{T}=\frac{\mathbf{1}_{n}^{T} \Sigma^{-1}}{\mathbf{1}_{n}^{T} \Sigma^{-1} \mathbf{1}_{n}}$. Since $\mathbf{E} C_{i}=\mathbf{E}\left(\boldsymbol{p}_{\boldsymbol{i}}^{T} \boldsymbol{X}\right)=\boldsymbol{p}_{\boldsymbol{i}}^{T} \mathbf{1}_{n} \mu=0$, $\boldsymbol{p}_{\boldsymbol{i}}^{T} \mathbf{1}_{n}=0$, while $i \in(0, n-1)$. Thus,

$$
\begin{gathered}
\boldsymbol{a}^{T} \Sigma \boldsymbol{p}_{\boldsymbol{i}}=\frac{\mathbf{1}_{n}^{T} \Sigma^{-1}}{\mathbf{1}_{n}^{T} \Sigma \mathbf{1}_{n}} \Sigma \boldsymbol{p}_{\boldsymbol{i}} \propto \mathbf{1}_{n}^{T} \boldsymbol{p}_{\boldsymbol{i}}=0 \\
P^{T} \Sigma \boldsymbol{a}=\mathbf{0}_{n}
\end{gathered}
$$

According to Appendix III, the covariance between $C_{i}$ and all leaf node values on the $(i+1)$-th tree are zero. Since $W_{j}(j>i)$ is a linear combination of leaf node values on the $(i+1)$-th tree, $\operatorname{Cov}\left(C_{i}, W_{j}\right)=0,(j \geqslant i)$. As a result, $\operatorname{Cov}\left(W_{n-1}, C_{i}\right)=0, i \in(0, n-1)$. Thus,

$$
\operatorname{Cov}\left(\boldsymbol{b}^{T} \boldsymbol{X}, \boldsymbol{p}_{\boldsymbol{i}}^{T} \boldsymbol{X}\right)=\boldsymbol{b}^{\boldsymbol{T}} \Sigma \boldsymbol{p}_{\boldsymbol{i}}=0, \quad i \in(0, n-1)
$$

$$
P^{T} \Sigma \boldsymbol{b}=\mathbf{0}_{n}
$$

Since $P$ is a $n$ by $n-1$ matrix with $\operatorname{rank}(P)=n-1$, and $\operatorname{rank}(\Sigma)=n, P^{T} \Sigma$ is a $n-1$ by $n$ matrix with $\operatorname{rank}\left(P^{T} \Sigma\right)=n-1$. With equations (27) and (26), $\boldsymbol{a}$ and $\boldsymbol{b}$ are linearly dependent to each other $(\boldsymbol{a} \| \boldsymbol{b}) . w_{n-1}$ and $\widehat{\mu}_{m l e}$ are proportional to each other. The scaling factor between $w_{n-1}$ and $\widehat{\mu}_{m l e}$ can be identified by normalizing their variance or expectation values. 


\section{APPENDIX V}

We show that the slope parameter of the ordinary least squares regression of OU-PIC conducted through the origin is identical to the slope parameter of generalized least squares regression under a OU model of evolution.

Proof. Recall that $c_{i}=\boldsymbol{p}_{\boldsymbol{i}}^{T} \boldsymbol{x}, \widehat{\mu}=\boldsymbol{a}^{T} \boldsymbol{x}$, and two matrices,

$$
P=\left[\begin{array}{ccc}
\mid & & \mid \\
p_{1}, & \cdots, & p_{n-1} \\
\mid & & \mid
\end{array}\right] \text { and } G=\left[\begin{array}{cccc}
\mid & \mid & \mid \\
p_{1}, & \cdots, & p_{n-1}, & a \\
\mid & & \mid & \mid
\end{array}\right] \text {. }
$$

We know that $P^{T} \Sigma P=\phi I_{n}$. According to Appendix IV, $P^{T} \Sigma \boldsymbol{a}=\mathbf{0}_{n}, \boldsymbol{a}^{T}=\frac{\mathbf{1}_{n}^{T} \Sigma^{-1}}{\mathbf{1}_{n}^{T \Sigma^{-1}} \mathbf{1}_{n}}$ and $\boldsymbol{a}^{T} \Sigma \boldsymbol{a}=\frac{1}{\mathbf{1}_{n}^{T \Sigma^{-1}} \mathbf{1}_{n}}$. As a result

$$
G^{T} \Sigma G=D, \quad D=\left[\begin{array}{cc}
\phi I_{n-1} & \mathbf{0}_{n-1} \\
\mathbf{0}_{n-1}^{T} & \frac{1}{\mathbf{1}_{n}^{T} \Sigma^{-1} \mathbf{1}_{n}}
\end{array}\right]
$$

We can derive that

$$
\begin{aligned}
\Sigma^{-1} & =G D^{-1} G^{T}=\left[\begin{array}{ll}
P & \boldsymbol{a}
\end{array}\right]\left[\begin{array}{cc}
\phi I_{n-1} & \mathbf{0}_{n-1} \\
\mathbf{0}_{n-1}^{T} & \mathbf{1}_{n}^{T} \Sigma^{-1} \mathbf{1}_{n}
\end{array}\right]\left[\begin{array}{c}
P^{T} \\
\boldsymbol{a}^{T}
\end{array}\right] \\
& =\phi P P^{T}+\frac{\Sigma^{-1} \mathbf{1}_{n} \mathbf{1}_{n}^{T} \Sigma^{-1}}{\mathbf{1}_{n}^{T} \Sigma^{-1} \mathbf{1}_{n}}
\end{aligned}
$$

The slope parameter of the ordinary least squares regression of OU-PIC through the origin is $\widehat{\beta}_{p i c}=\frac{\boldsymbol{x}^{T} P P^{T} \boldsymbol{y}}{\boldsymbol{x}^{T} P P^{T} \boldsymbol{x}}$.

We next find the solution for the generalized least squares problem, $y=\beta_{0}+\beta_{1} x$, with residual distribution $\epsilon \sim N(0, \Sigma)$.

$$
\left[\begin{array}{c}
\widehat{\beta}_{0} \\
\widehat{\beta}_{1}
\end{array}\right]=\left(X^{T} \Sigma^{-1} X\right)^{-1} X^{T} \Sigma^{-1} \boldsymbol{y}, \quad X=\left[\begin{array}{ll}
\mathbf{1}_{n} & \boldsymbol{x}
\end{array}\right]
$$

We can solve

$$
\begin{aligned}
\widehat{\beta}_{1} & =\frac{\left(\mathbf{1}_{n}^{T} \Sigma^{-1} \mathbf{1}_{n}\right)\left(\boldsymbol{x}^{T} \Sigma^{-1} \boldsymbol{y}\right)-\left(\boldsymbol{x}^{T} \Sigma^{-1} \mathbf{1}_{n}\right)\left(\mathbf{1}_{n}^{T} \Sigma^{-1} \boldsymbol{y}\right)}{\left(\mathbf{1}_{n}^{T \Sigma^{-1}} \mathbf{1}_{n}\right)\left(\boldsymbol{x}^{T} \Sigma^{-1} \boldsymbol{x}\right)-\left(\boldsymbol{x}^{T} \Sigma^{-1} \mathbf{1}_{n}\right)\left(\mathbf{1}_{n}^{T \Sigma^{-1}} \boldsymbol{x}\right)} \\
& =\frac{\boldsymbol{x}^{T}\left[\left(\mathbf{1}_{n}^{T} \Sigma^{-1} \mathbf{1}_{n}\right) \Sigma^{-1}-\Sigma^{-1} \mathbf{1}_{n} \mathbf{1}_{n}^{T} \Sigma^{-1}\right] \boldsymbol{y}}{\boldsymbol{x}^{T}\left[\left(\mathbf{1}_{n}^{T} \Sigma^{-1} \mathbf{1}_{n}\right) \Sigma^{-1}-\Sigma^{-1} \mathbf{1}_{n} \mathbf{1}_{n}^{\left.T \Sigma^{-1}\right]} \boldsymbol{x}\right.}
\end{aligned}
$$


Plug in equation (28) to $\widehat{\beta}_{1}$, we have

$$
\widehat{\beta}_{1}=\frac{\boldsymbol{x}^{T} P P^{T} \boldsymbol{y}}{\boldsymbol{x}^{T} P P^{T} \boldsymbol{x}}=\widehat{\beta}_{p i c} .
$$

